\title{
Inverse micellar sugar glass (IMSG) nanoparticles for transfollicular vaccination
}

Ankit Mittal $^{1 \#}$, Kai Schulze ${ }^{2 \#}$, Thomas Ebensen ${ }^{2}$, Sebastian Weissmann $^{2}$, Steffi Hansen ${ }^{1,3}$, Carlos A. Guzmán², *, Claus-Michael Lehr ${ }^{1,3, *}$

\footnotetext{
${ }^{1}$ Saarland University, Biopharmaceutics and Pharmaceutical Technology, Saarbruecken, Germany

${ }^{2}$ Helmholtz Center for Infection Research (HZI), Department of Vaccinology and Applied Microbiology, Braunschweig, Germany

${ }^{3}$ Helmholtz Institute for Pharmaceutical Research Saarland (HIPS), Helmholtz Centre for Infection Research (HZI), Saarland University, Saarbruecken, Germany

\# These authors contributed equally to the manuscript

* Shared corresponding authorship:
}

Claus-Michael Lehr, Helmholtz Institute for Pharmaceutical Research Saarland (HIPS), Helmholtz Centre for Infection Research (HZI), Saarland University, Saarbruecken, Germany; Email: claus-michael.lehr@helmhotz-hzi.de; Tel.: ++49 (681) 302 3039; fax: ++49 (681) 3024677

Carlos A. Guzmán, Helmholtz Centre for Infection Research (HZI), Braunschweig, Germany; Email: cag@helmholtz-hzi.de; Tel.: ++49 (531) 6181 4600; fax: ++49 (531) 61814699 


\begin{abstract}
Transfollicular antigen delivery through the intact skin is an interesting new avenue for needle-free vaccination. The aim of this work was to evaluate the potential of surfactant based inverse micellar sugar glass nanoparticles (IMSG NPs) as a delivery system for such purpose. To this end, we evaluated the strength and type of immune response elicited after administration of IMSG NPs containing the model antigen ovalbumin (OVA) by intranasal, transfollicular or intradermal route. Furthermore, we explored the possibility of improving the immune response elicited by coencapsulating the adjuvant bis-(3',5')-cyclic dimeric adenosine monophosphate (c-diAMP) and OVA within one particulate carrier system.
\end{abstract}

The study showed enhanced stability and encapsulation efficacy of the antigen when encapsulated in IMSG NPs in comparison to polylactic-co-glycolic acid (PLGA) and chitosan-PLGA NPs. Moreover, by transfollicular delivery, IMSG NPs showed enhanced follicular uptake in comparison to OVA solution or OVA-loaded chitosanPLGA NPS.

While the immune response stimulated after intranasal administration was negligible, significant humoral and cellular responses were observed after immunization via transfollicular and intradermal route. This holds particularly true when OVA and c-diAMP were co-encapsulated in IMSG NPs, as compared to OVA \pm c-di-AMP solution or OVA-loaded IMSG NPs without adjuvantation. The results of this study underscore the potential of transfollicular vaccination, but also the need for optimized nanocarriers and adjuvants.

Keywords: inverse micellar sugar glass nanoparticles, vaccination, transfollicular, ovalbumin

\title{
1. Introduction
}

Although vaccination is considered as one of the most effective medical interventions introduced in human history, new safe, efficient, minimally invasive and cheap vaccination methods are needed to meet the current challenges in vaccine delivery (such as lack of compliance, safety issues, logistic constraints, etc.) [1]. Among the main vaccination methods (i.e. live-attenuated, inactivated and subunit vaccines), subunit vaccines are considered the safest. However, subunit vaccines generally suffer from poor immunogenicity [2]. Novel delivery systems and/or alternative routes for administration may be keys to address this roadblock.

A common strategy to improve immunogenicity of subunit antigens is their encapsulation into a particulate carrier. Such carriers not only enhance immunogenicity by mimicking the size of microorganisms, but they also help by stabilizing protein antigens, ensuring their consistent release, enhancing antigen uptake by antigen presenting cells (APCs) and allowing co-encapsulation of adjuvants. This results in increased antigen-specific immune responses with potentially reduced amounts of antigen needed [3].

Polymeric nanoparticles (NPS) such as polylactic-co-glycolic acid (PLGA), polylactic acid (PLA) and chitosan-PLGA (Chit-PLGA) have been extensively studied as antigen delivery systems [4]. To this end, emulsion based methods are often applied to encapsulate antigens into polymeric NPs. However, these particles still showed some drawbacks in terms of limited antigen loading capacity and incomplete release of encapsulated antigen. Furthermore, during the NP preparation process, proteinbased payloads may be exposed to several process- and storage-related stresses (such as liquid/liquid and solid/liquid interfaces), which result in significant 
degradation, loss of bioactivity and safety concerns, such as suboptimal immune responses [5-7]. Recently, Giri et al. showed a generic approach for efficient incorporation and stabilization of proteins for tissue engineering and drug delivery using principles from enzyme chemistry and bio-pharmaceutics [8]. In this approach, proteins were protected from nonpolar solvent exposure using an inverse micelle technique, i.e. by dispersing protein into an organic solution of surfactants and subsequently freeze drying them before introducing them into polymer solutions for tissue engineering.

Administration of antigen solutions and particulate carriers by intradermal (ID) and intranasal (IN) routes has been widely studied [3, 9]. However, recent attention focuses on non-invasive transcutaneous vaccination strategies. One of the main difficulties is that antigen solutions and particulate carriers cannot penetrate the superficial layers of the intact skin or even partially damaged skin, due to the formidable stratum corneum (SC) barrier. Thus, it is highly unlikely to reach the viable cells of the epidermis or beyond [10]. However, studies made by Mahe et al. showed that particles of a size of approx. $200 \mathrm{~nm}$ applied to the skin were taken up by the Langerhans cells (LCs) located around the hair follicles [11]. Our previous studies explored the transfollicular (TF) route as a potential immunization route using ovalbumin (OVA) loaded Chit-PLGA NPs co-admixed with c-di-AMP as adjuvant. The obtained results showed the stimulation of efficient immune responses without disrupting the skin by using nano-carriers in combination with an appropriate adjuvant $[12,13]$. Nonetheless, in order to further improve follicular penetration as well as overcoming the already mentioned obstacles of PLGA particles, antigen-loaded IMSG NPs with and without co-encapsulated adjuvant were explored as potential vaccine delivery systems for TF administration.

To this end, we prepared antigen-loaded NPs by efficient incorporation and stabilization of antigen using an inverse micellar technique and pharmaceutical excipients (i.e. surfactants, sugars and oil generally regarded as safe and GRAS listed). These IMSG NPs were finally dispersed in an oily dispersion (medium-chain triglycerides, which is broadly used for parenteral nutrition, as a readily digestible energy source for the neonate, for cosmetics, in diet programs and as solvents for flavors and oral medicines and vitamins [14].

IMSG NPs were characterized physico-chemically in terms of size, surface charge and morphology, antigen integrity and biological activity. To assess if incorporation of an adjuvant would further enhance the efficacy of the vaccine formulation, bis- $\left(3^{\prime}, 5^{\prime}\right)$ cyclic dimeric adenosine monophosphate (c-di-AMP) was co-encapsulated. The immunogenicity of the formulations was evaluated in vivo immunizing mice via TF, IN and ID route.

\section{Materials and methods}

\subsection{Mice}

C57BL/6 (H-2b) female mice (6-8 weeks old) were purchased from Harlan (Germany). OVA-TCR transgenic mice C57BL/6-Tg(TcraTcrb)1100Mjb/J (OT-I) and C57BL/6-Tg(TcraTcrb)425Cbn (OT-II) were bred at the animal facility of the Helmholtz Centre for Infection Research (Germany) under specific pathogen-free conditions. All animal experiments presented in this study have been performed with the ethical agreement of the local government of Lower Saxony (Germany) under the permit No. 33.11.42502-04-017/08. 


\subsection{Nanoparticle preparation and characterization}

\subsubsection{Nanoparticle preparation}

IMSG NPs were prepared from inverse micelles of lecithin in chloroform. $440 \mathrm{mg}$ of lecithin and $60 \mathrm{mg}$ span 80 were dissolved in $1.4 \mathrm{ml}$ of chloroform in a glass vial using continuous stirring. Aqueous phase of $0.15 \mathrm{ml}$ containing OVA and trehalose $(6$ $\mathrm{mg}$ ) was then added. The mixture was then sonicated for $20 \mathrm{~s}$ at $8 \mathrm{~W}$. The inverse micelle suspension was flash-frozen in liquid $\mathrm{N}_{2}$ and subsequently freeze dried for 48 h. The resulting particles were dispersed in medium chain triglycerides (MCT).

For co-encapsulation of OVA and c-di-AMP in IMSG NPs, c-di-AMP was added to the aqueous phase. Vaccine formulations for the TF route contained OVA $(6 \mathrm{mg})$ and cdi-AMP $(0.9 \mathrm{mg})$. The ID and IN route formulations contained OVA $(2 \mathrm{mg})$ and c-diAMP (1.5 mg).

FITC labelled IMSG NPs were prepared by replacing OVA with FITC-OVA. OVAloaded Chit-PLGA NPs were prepared by a modified double emulsion method, as previously described [12]. See supplementary materials for additional details.

\subsubsection{Nanoparticle characterization}

Size and $\zeta$-potential of the NPs were analyzed by photon correlation spectroscopy (PCS) using a Nano-ZS (Malvern Instruments, Malvern, UK). The morphology of the NPs was characterized using transmission electron microscopy (TEM) (Tecnai 12 G2, FEI Company Hillsboro, Oregon, USA). For TEM, prior to scanning diluted suspensions of freeze dried NPs in cyclohexane were taken and mounted on a copper grid and subsequently dried at room temperature. Then, samples were negatively stained with a $1 \%$ solution of phosphor-tungstic acid and further examined. The integrity and bio-availability of the encapsulated OVA was monitored by SDS page and ELISA (more details are in the supplementary materials section).

\subsubsection{Follicular uptake of OVA-loaded IMSG NPs using excised pig ears: Influence of encapsulating OVA into particles}

In order to validate the successful penetration of IMSG NPs into hair follicles by TF vaccination, proof of concept studies quantifying follicular uptake were conducted as previously described [12], see supplementary materials for additional details.

\subsection{Immunization protocols}

Female C57BL/6 mice $(n=5)$ were immunized on day 0,14 and 28 with different vaccine formulations encompassing either OVA protein alone or admixed with c-diAMP in an aqueous solution or encapsulated in IMSG NPs via the IN, ID or TF route. Prior to the vaccination studies via the TF route, proof of concept studies were performed using an adoptive transfer model based on OT-II mice derived OVAspecific TCR-transgenic T cells (see suppl. Section 1.6). Antigen dose and application volumes were adjusted to the specific administration route, as depicted in Table 1. 
Table 1

Immunization groups

\begin{tabular}{ccccc}
\hline Group & Route & Dose (OVA) & $\begin{array}{c}\text { Dose } \\
(\text { c-di-AMP) }\end{array}$ & $\begin{array}{c}\text { Volume } \\
(\mu \mathrm{l})\end{array}$ \\
\hline Blank NPs + c-di-AMP & IN & - & $7.5 \mu \mathrm{g}$ & 20 \\
OVA & IN & $10 \mu \mathrm{g}$ & - & 20 \\
OVA + C-di-AMP & IN & $10 \mu \mathrm{g}$ & $7.5 \mu \mathrm{g}$ & 20 \\
OVA NPs & IN & $10 \mu \mathrm{g}$ & - & 20 \\
OVA + C-di-AMP NPs & IN & $10 \mu \mathrm{g}$ & $7.5 \mu \mathrm{g}$ & 20 \\
Blank NPs + c-di-AMP & ID & - & $7.5 \mu \mathrm{g}$ & 20 \\
OVA & ID & $10 \mu \mathrm{g}$ & - & 20 \\
OVA + C-di-AMP & ID & $10 \mu \mathrm{g}$ & $7.5 \mu \mathrm{g}$ & 20 \\
OVA NPs & ID & $10 \mu \mathrm{g}$ & - & 20 \\
OVA + C-di-AMP NPs & ID & $10 \mu \mathrm{g}$ & $7.5 \mu \mathrm{g}$ & 20 \\
Blank NPs + C-di-AMP & TF & - & $7.5 \mu \mathrm{g}$ & 60 \\
OVA & TF & $200 \mu \mathrm{g}$ & - & 60 \\
OVA + C-di-AMP & TF & $200 \mu \mathrm{g}$ & $20 \mu \mathrm{g}$ & 60 \\
OVA NPs & TF & $200 \mu \mathrm{g}$ & - & 60 \\
OVA + c-di-AMP NPs & TF & $200 \mu \mathrm{g}$ & $20 \mu \mathrm{g}$ & 60 \\
\hline
\end{tabular}

ID: intradermal, IN: intranasal, TF: transfollicular application of NPS

TF vaccination was conducted on the flank part of the mice. In brief, 2 days prior to immunization the mice were anesthetized and the hair was removed at the side for immunization using clippers and a depilatory cream. Depilated areas were washed and carefully inspected for cuts or skin irritation, in which case these mice were excluded from the experiment. For ID application mice were shaved to remove the hair at the side of injection and the vaccine formulation was injected holding the needle nearly parallel to the plane of the skin. IN immunizations were performed as described earlier [15].

\subsection{Sample collection}

Blood samples from immunized mice were taken from the retro-orbital complex on day $-1,13,27$ and 48 . To separate sera, blood samples were incubated for $1 \mathrm{~h}$ at $37^{\circ} \mathrm{C}$ followed by 30 min incubation at $4^{\circ} \mathrm{C}$. Subsequently, samples were centrifuged at $3000 \times \mathrm{g}$ for $10 \mathrm{~min}$ and sera were stored at $-20^{\circ} \mathrm{C}$ until further processing. On day 48, mice were sacrificed, draining lymph nodes (LNs; cervical in case of IN and inguinal in case of ID and TF immunization) and spleens were collected and single cell suspensions were obtained by pressing the organs through a mesh (cell strainer Falcon 2340) with the help of a sterile syringe plunger. Cell suspensions were generated and analyzed for the presence of antigen-specific cells, as previously described [15].

\subsection{Detection of antigen-specific antibodies}

OVA-specific antibodies in sera of individual animals were determined by ELISA using microtiter plates coated with $100 \mu \mathrm{l} / \mathrm{well}$ of the antigen $(2 \mu \mathrm{g} / \mathrm{ml}$ in $0.05 \mathrm{M}$ carbonate buffer, $\mathrm{pH}$ 9.6), as previously described [15] (for more details see supplementary methods section).

\subsection{Evaluation of cellular proliferation and cytokine profiles}

The proliferative activity of immune cells derived from the spleen and LNs of immunized mice as well as their cytokine profiles were measured as previously described [15], for additional details see the supplementary methods section. 


\subsection{Multifunctional T cells}

To evaluate the capacity of different vaccine formulations to stimulate OVA-specific multifunctional $T$ cells, splenocytes from immunized mice were isolated and their capacity to produce different cytokines was evaluated by flow cytometry (more details are in the supplementary methods section).

\subsection{Statistical analysis}

Statistical significance of the observed differences was analyzed using the nonparametric ANOVA analyses with Kruskal-Wallis test of the Graph Pad Prism 5 software for Windows (Version 5.04). Differences were considered significant at $\mathrm{p}<0.05\left(^{*}\right), p<0.01\left(^{* \star}\right)$ and $p<0.001\left(^{* * *}\right)$, respectively.

\section{Results}

\subsection{Characterization of OVA-loaded IMSG NPS}

The characteristics of the NPs are summarized in Table 2. The TEM image in Fig.1 shows smooth, spherical particles. The mean size of OVA-loaded IMSG NPs (+/- cdi-AMP) regardless of the added amount of OVA (2 mg or $6 \mathrm{mg}$ ) and c-di-AMP (none, $0.9 \mathrm{mg}$, or $1.5 \mathrm{mg}$ ) was approximately $300-350 \mathrm{~nm}$ with a mono-disperse size distribution (PDI < 0.2). The encapsulation efficiency of OVA and c-di AMP in IMSG NPs is $100 \%$. Adjuvant incorporation into NPs did not change the particle characteristics in terms of size and PDI.

Table 2.

Physico-chemical characterization of the optimized IMSG NPs

\begin{tabular}{lllll}
\hline S.no. & NPs & Route & Size $(\mathbf{n m})$ & PDI \\
\hline 1 & OVA-loaded IMSG NPs & ID and IN & $312.2 \pm 14.6$ & $0.146 \pm 0.02$ \\
2 & OVA + c-di-AMP IMSG NPs & ID and IN & $301.1 \pm 14.2$ & $0.146 \pm 0.01$ \\
3 & OVA-loaded IMSG NPs & TF & $317.4 \pm 22.1$ & $0.125 \pm 0.04$ \\
4 & OVA + c-di-AMP IMSG NPs & TF & $323.6 \pm 9.4$ & $0.132 \pm 0.03$ \\
\hline \multicolumn{7}{l}{ Values } & represent mean \pm standard & deviation of & $\mathrm{n}=6$ independently & prepared \\
batches. PDI: poly-dispersity index & & &
\end{tabular}

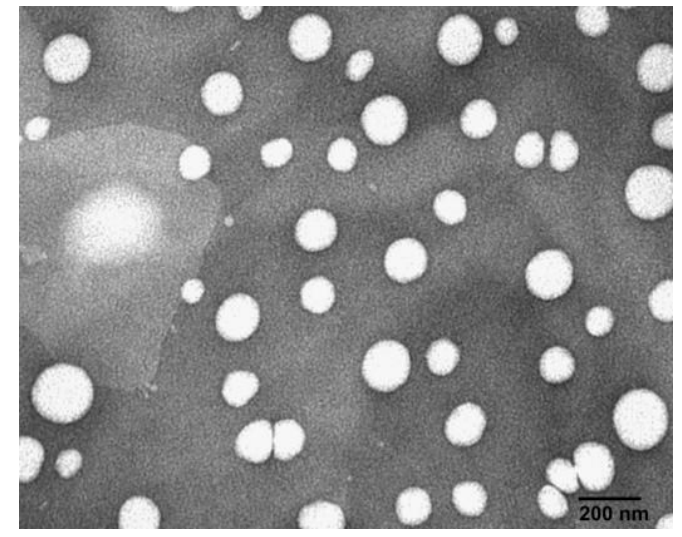

Fig. 1. TEM image of OVA-loaded IMSG NPs

\subsection{Intranasal vaccination}

\subsubsection{Adjuvanted IMSG NPs fail to stimulate efficient humoral immune responses following IN immunization}

Serum anti-OVA IgG antibody titers obtained after IN vaccination with different formulations are shown in Fig. 2A. The OVA + c-di-AMP solution stimulated the highest OVA-specific IgG titers as compared to OVA+ C-di AMP loaded IMSG NPs $(p<0.05)$ and OVA loaded IMSG NPs $(p<0.01)$. Surprisingly, OVA-loaded IMSG NPs 
and OVA + c-di-AMP co-encapsulated NPs via the IN route stimulated no or only very low IgG titers. Moreover, addition of $\mathrm{c}$-di-AMP to the OVA solution significantly increased $(p<0.01)$ the production of IgG $2 c$ antibodies compared to all other tested formulation (Fig. 2C).

\subsubsection{Adjuvanted IMSG NPs fail to stimulate efficient cellular immune responses following IN immunization}

IN immunization of mice with IMSG NPs did not result in the stimulation of strong cellular responses. Only marginal proliferative capacities of splenocytes and LN cells derived from mice vaccinated with OVA + c-di-AMP co-encapsulated IMSG NPs were observed (Fig. 3A). In contrast, strong proliferative responses were observed when cells from control mice receiving OVA solution co-administered with c-di-AMP were tested (Fig. 3A). These observations were further validated by the levels of cytokine production obtained following IN immunization (Fig. 3B). Thus, no or only very low amounts of cytokines were produced by cells from mice receiving OVA-loaded IMSG NPs and OVA + c-di-AMP co-encapsulated IMSG NPs, except for IFN $\gamma$ which was produced to a higher extent by cells from animals receiving these particular formulations (Fig. 3B).

Recent findings support the assumption that vaccine efficacy not only depends on the strength but also, to a large extent, on the quality of the stimulated $T$ cell response. In this regard, stimulation of multifunctional $T$ cells which possess a broader functional spectrum results in enhanced protective immunity, as compared to mono-functional cells [11]. Therefore, we investigated the quality of the $T$ cell responses stimulated by IMSG NPs by flow cytometry. 


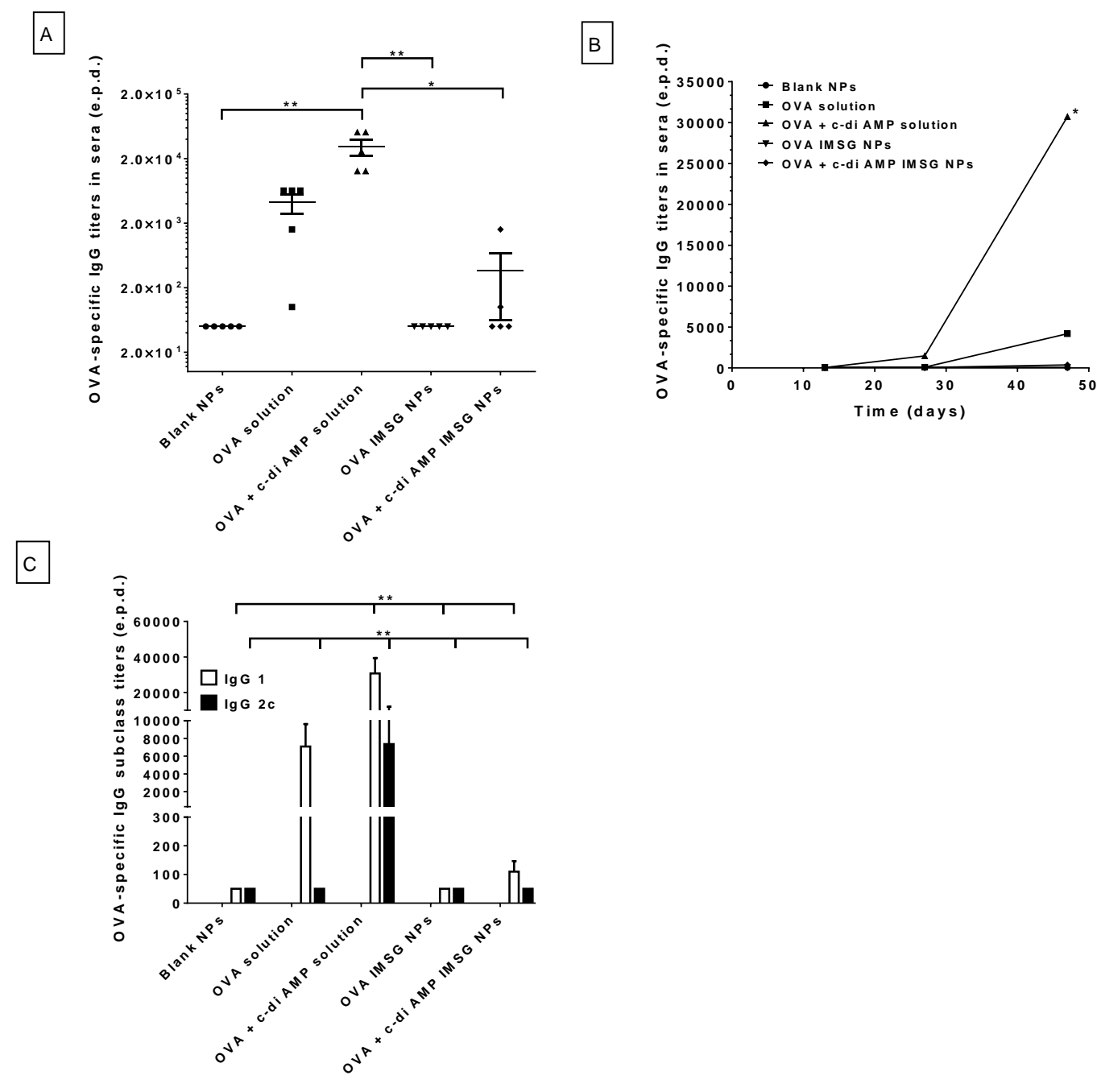

Fig. 2. Systemic humoral immune responses stimulated in C57BL/6 mice $(n=5)$ after three immunizations with different OVA containing formulations via the intranasal (IN) route. (A) OVAspecific IgG titers in sera of mice 20 days after the last immunization. Bullet points indicate titers for individual animals and horizontal lines the mean for each experimental group. (B) Kinetics of OVAspecific IgG production in sera of immunized mice on day 0, 13, 27 and 48. (C) OVA-specific IgG subclasses in sera of immunized mice. Results are expressed as the last dilution (end point dilution, e.p.d.) giving the double value (OD450 nm) of the background value (negative control). Standard error of mean (SEM) is indicated by vertical lines. Differences between the group receiving OVA + C-di-AMP solution and all other tested groups were considered significant at $p<0.05\left(^{*}\right)$, and $p<0.01 \quad\left(^{* *}\right)$, respectively.

None of the vaccine formulations were able to stimulate antigen-specific CD8+ T cells when applied by IN route (data not shown). However, CD4+ cellular responses were slightly increased in mice vaccinated with OVA + c-di-AMP co-encapsulated in IMSG NPs (Fig. 3C). Thus, incorporation of c-di-AMP resulted in higher numbers of OVA-specific bi-functional (IFN $\gamma+/ T N F a+, \quad$ IFN $\gamma+/ / L-2+)$ and tri-functional (IFN $\gamma+/ T N F a / / L-2+) ~ C D 4+T$ cells, as compared to values in animals receiving OVA alone and OVA-encapsulated IMSG NPs, respectively (Fig. 3C). Control mice receiving OVA + c-di-AMP solution showed the highest number of IFN $\gamma$ producing CD4+ T cells and a slightly higher proportion of bi- and tri-functional cells (Fig. 3C). 
A
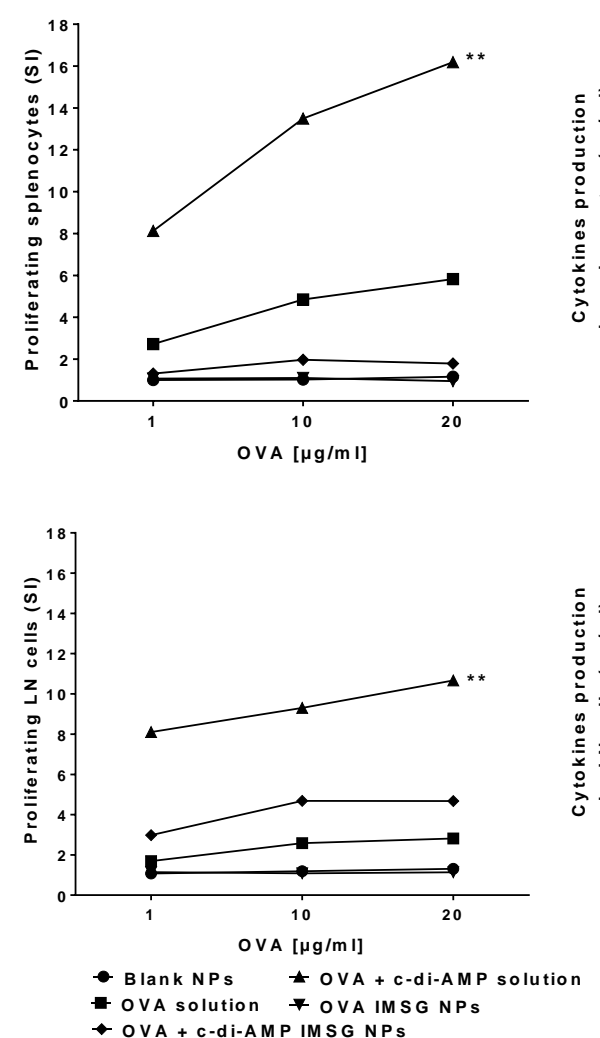

$\rightarrow$ OVA + c-di-AMP IMSG NPs
B
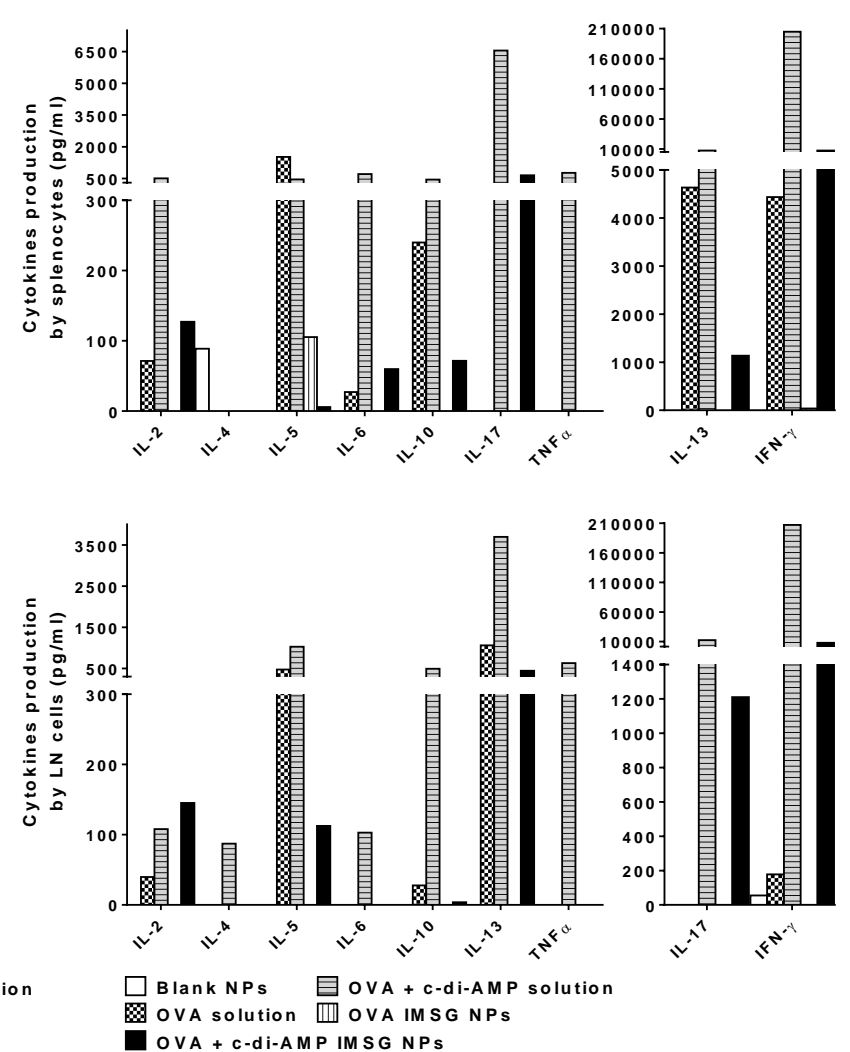

$\%$ of IFNg+ CD4+ cells

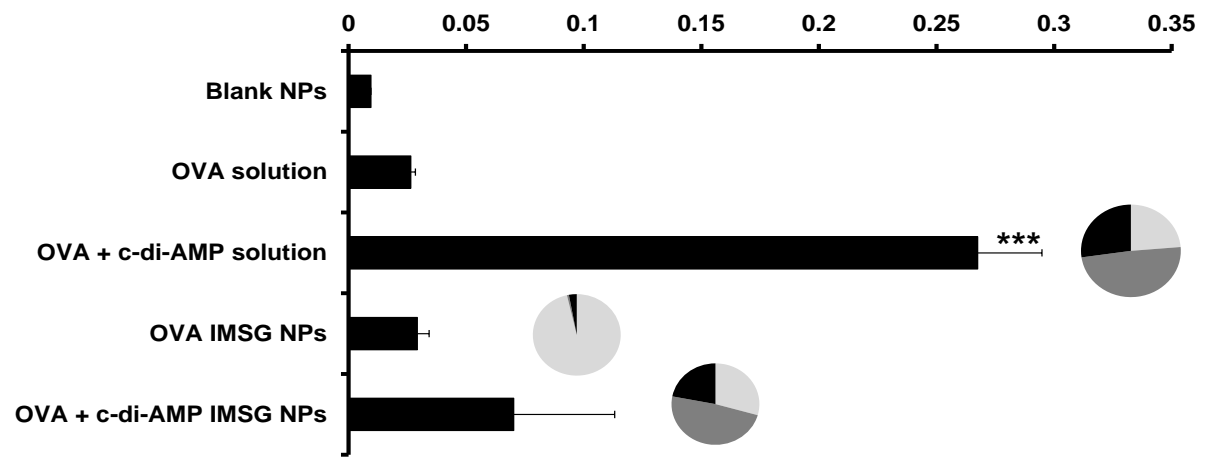

Fig. 3. Cellular responses stimulated following IN immunization. (A and B) Splenocytes (upper panel) and $\mathrm{LN}$ cells (lower panel) from vaccinated animals were restimulated with different concentrations of OVA for $96 \mathrm{~h}$. (A) Cellular proliferation was assessed by determination of the $\left[{ }^{3} \mathrm{H}\right]$ thymidine incorporated into the DNA of replicating cells. Results are averages of quadruplicates and expressed as stimulation index ( $\mathrm{SI})$. (B) Cytokine profiles stimulated by the different vaccine formulations were evaluated by cytometric bead array. Results are expressed in $\mathrm{pg} / \mathrm{ml}$. (C) Quality of the $\mathrm{T}$ cell responses stimulated following IN vaccination. Results are expressed as \% of all CD4+ cells acquired per sample (approx. 130,000 CD4+ cells). This subpopulation was further divided into mono-functional (IFN $\gamma$ positive) (light gray), bi-functional (IFN $\gamma /$ IL-2 or IFN $\gamma /$ TNF $\alpha$ positive) (dark gray) and trifunctional cells (IFN $\gamma$, IL-2 and TNF $\alpha$ positive) (black). Differences were considered significant as compared to values in mice receiving OVA alone at $p<0.01\left(^{* *}\right)$ and $p<0.001\left(^{* * *}\right)$, respectively.

Beside IFN $\gamma$ single producer cells, we also observed TNF $\alpha$ single producers, whereas almost no IL-2 single producer cells were detected, independently of the route of administration. This is in line with the proliferative capacities obtained after restimulation of spleen and LN derived cells, since TNF $\alpha$ enhances the proliferation 
of $T$ cells induced by various stimuli in the absence of IL-2. We also observed higher numbers of bi-functional CD4+ cells expressing IFN / TNF $\alpha$ and IL-2 / TNF $\alpha$, compared to IFN / IL-2 double producer cells (data not shown). This is not surprising, as IL-2 expression is most often associated with TNF $\alpha$ expression rather than IFN $\gamma$ (reviewed in [16]). T cells which produce both IFN $\gamma$ and TNF $\alpha$ were shown to mediate more efficient killing as compared to cells producing each of the other cytokines alone, whereas those expressing IL-2 / TNF $\alpha$ seem to serve as a reservoir for memory CD4+ cells (reviewed in [16]).

\subsection{Transfollicular vaccination}

The feasibility of TF vaccination was evaluated prior to the immunization studies by investigating in vitro the follicular uptake and by performing an in vivo adoptive transfer experiment (suppl. section 1.6). In brief, mice were injected CFSE-labeled OVA-specific TCR transgenic CD4+ T cells prior to TF administration to increase the number of antigen-specific T cells. Mice were sacrificed $96 \mathrm{~h}$ after vaccination, splenocytes were isolated and the proliferative capacity of the transgenic $T$ cells was analyzed by FACS. A positive proliferation confirmed that the antigen administered by the TF route was taken up, processed and presented to the antigen-specific $T$ cells., thereby demonstrating the feasibility of the IMSG NPs-based TF vaccination approach (see suppl. Section 1.6).

\subsubsection{Follicular uptake of OVA loaded IMSG NPs: Influence of encapsulating OVA into particles}

In contrast to the immunization studies, in this experiment we used FITC-conjugated OVA (in solution or encapsulated into NPs) to evaluate the follicular uptake of OVA loaded IMSG NPs. Fig. 4(A,B,C) shows representative images illustrating the distribution of fluorescently labeled IMSG NPs on the skin surface as well as in the hair follicle. NPs accumulate in the follicle openings, cover the hair and invade the follicular duct.

The magnitude of NPs invaded was evaluated by quantifying the fluorescence measured in cyanoacrylate biopsy extracts (Fig. 4D). In the case of OVA solution only $0.8 \%-1.5 \%\left(0.13 \mu \mathrm{g} \pm 0.04 \mu \mathrm{g} / \mathrm{cm}^{2}\right)$ of the applied antigen $\left(11.3 \mu \mathrm{g} / \mathrm{cm}^{2}\right)$ entered the hair follicle. When using Chit-PLGA NPs, this amount has already increased to $2.2 \%-3.4 \%\left(0.31 \mu \mathrm{g} \pm 0.07 \mu \mathrm{g} / \mathrm{cm}^{2}\right)$ whereas the application of IMSG NPs further increased the amount of OVA delivered to the hair follicle to $4.3 \%-6.3 \%(0.58 \mu \mathrm{g} \pm$ $\left.0.11 \mu \mathrm{g} / \mathrm{cm}^{2}\right)$. Thus, encapsulation of OVA into IMSG NPs significantly enhanced $(p<0.001)$ follicular delivery of OVA by a factor of $4.38 \pm 0.81$ compared to OVA solution and $1.89 \pm 0.35$ compared to OVA-loaded Chit-PLGAs.
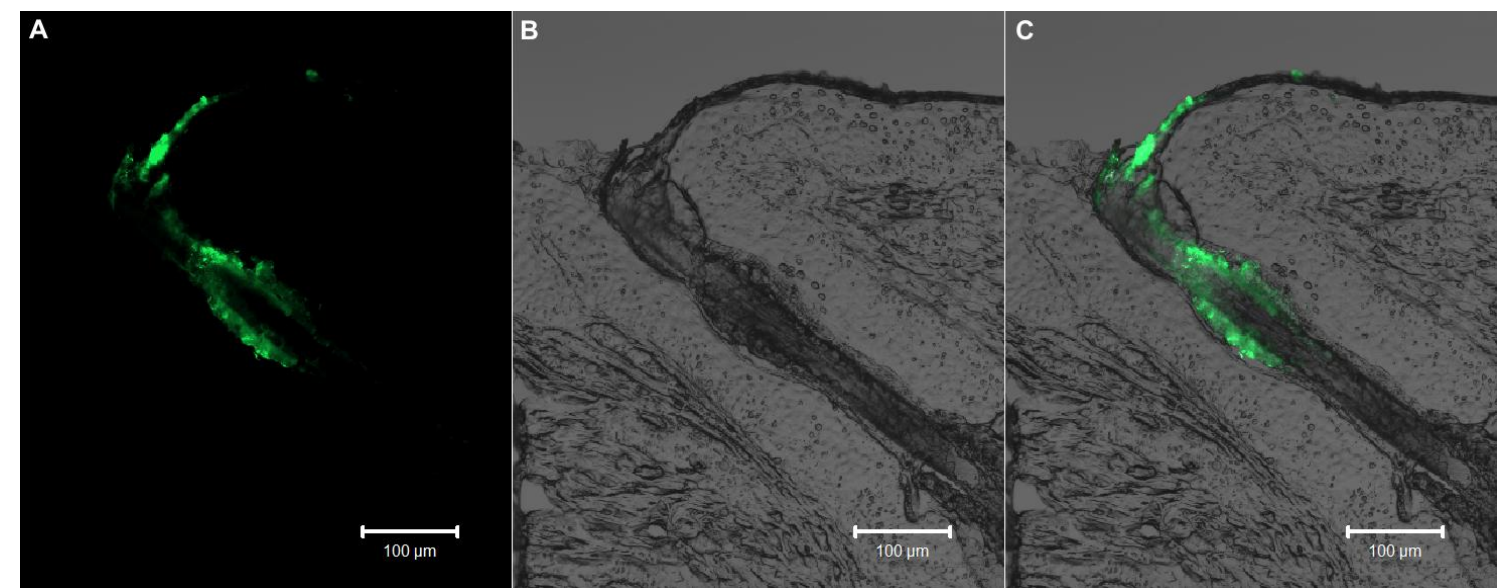


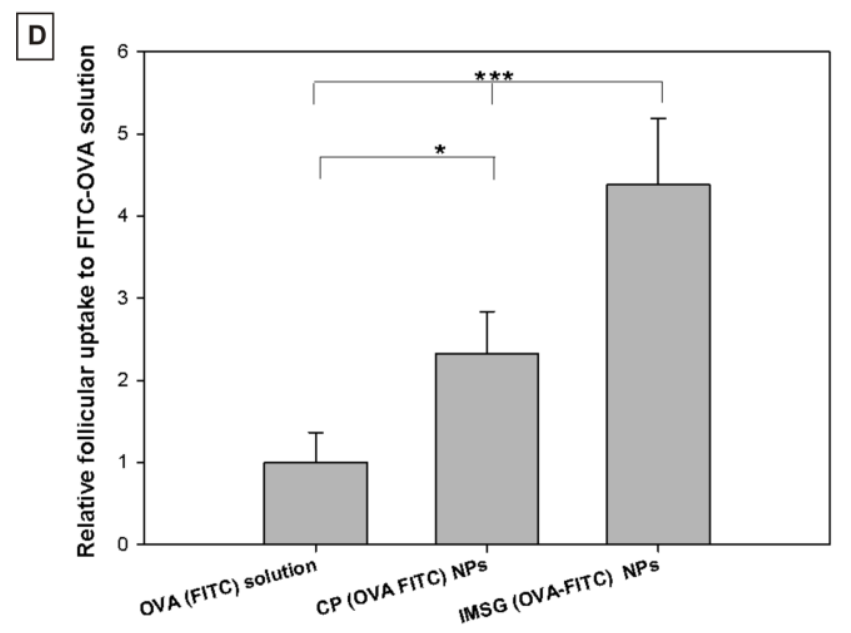

Fig. 4. A) Distribution of fluorescently labeled NPs in hair follicles after application to excised pig ears. (LSM 510 Meta, Carl Zeiss GmbH, Jena Germany; excitation wavelength $458 \mathrm{~nm}$ ), Transversal cryosection (thickness $12 \mu \mathrm{m})(B)$; overlay of fluorescence and transmission light images (C): NPs are visible on the skin surface and inside the follicle. (D) Uptake of FITC-labeled OVA encapsulated in IMSG NPs relative to FITC-OVA solution and FITC-OVA in CP NPs. $(n=6$, mean \pm SD; differences were considered significant whenever $p<0.05\left({ }^{*}\right), p<0.001\left(^{(* \star}\right)$ compared to OVA-FITC solution).

\subsubsection{Adjuvanted IMSG NPs stimulate efficient humoral immune responses following TF vaccination}

In contrast to IN vaccination, when mice were vaccinated by the TF route, OVAloaded IMSG NPs or OVA + c-di-AMP co-encapsulated IMSG NPs stimulated significantly stronger $\operatorname{lgG}$ production than OVA protein alone or, although not significantly, co-administered with c-di-AMP. This indicates an enhanced delivery efficacy of the antigen upon encapsulation into IMSG NPs. Moreover, the highest anti-OVA IgG titers were observed in serum of mice receiving OVA + c-di-AMP coencapsulated in IMSG NPs (Fig. 5A), as compared to OVA solution $(p<0.05)$ and blank $(p<0.001)$, thereby showing the beneficial effect of co-encapsulating adjuvant and antigen in the same NP. Moreover, mice receiving OVA + c-di-AMP coencapsulated in IMSG NPs generated significantly higher $(p<0.01)$ IgG titers compared to groups receiving OVA solutions, even after the $1^{\text {st }}$ boost (Fig. 5B). Surprisingly, none of the tested formulations following TF immunization promoted the elicitation of IgG2C antibodies (Fig.5C). This is in contrast to our previous study where mice were immunized via TF route using chitosan conjugated PLGA NPs, which stimulated production of IgG2c antibodies [13], as well as previous vaccination studies in which c-di-nucleotides were used as adjuvants for soluble antigens ([15, 17-20]). 


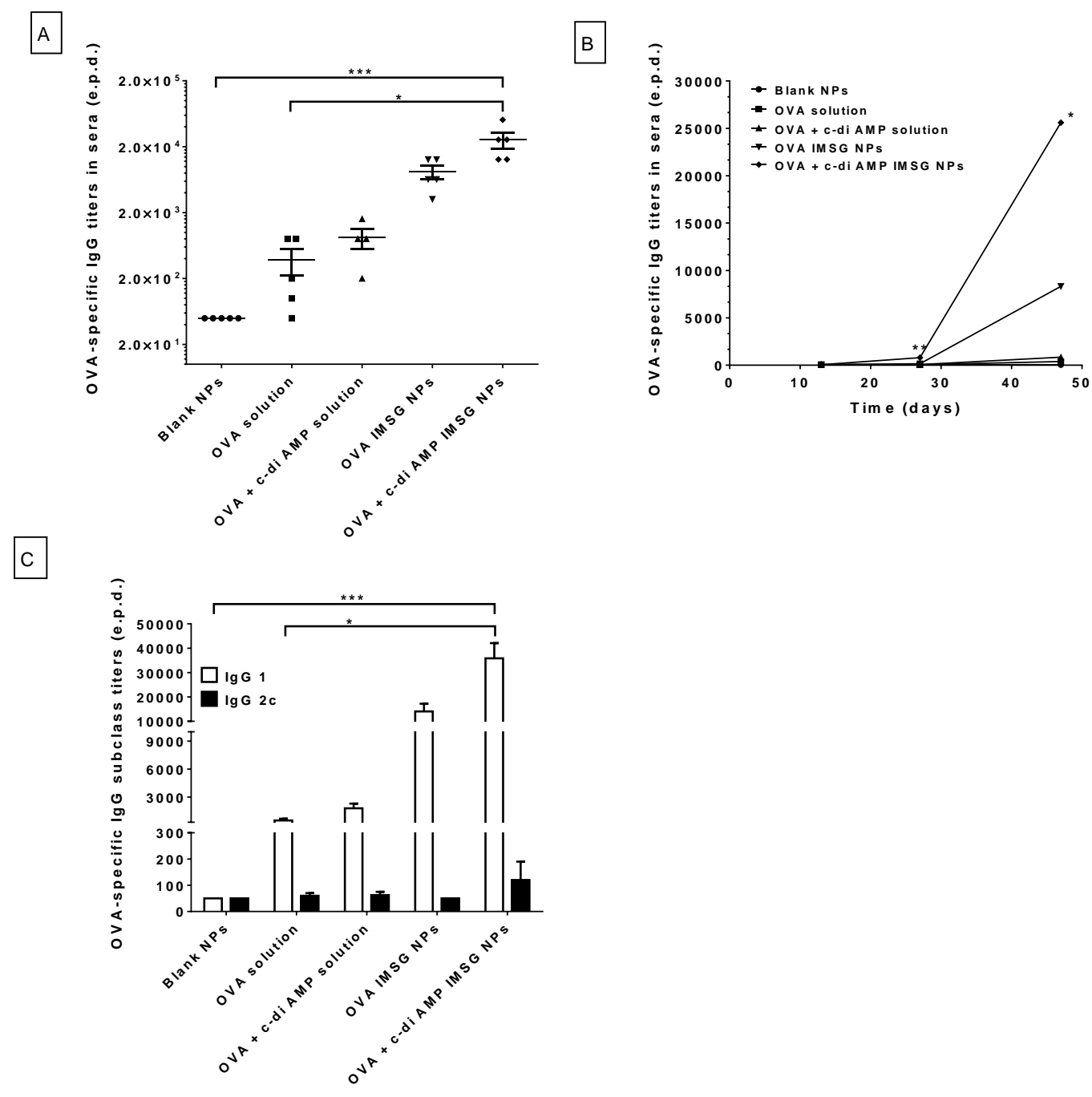

Fig. 5. Humoral immune responses stimulated in C57BL/6 mice after three vaccinations with OVA containing formulations by TF route. (A) Total OVA specific IgG titers in sera after immunization $(n=$ 5). Bullet points indicate titers for individual animals and horizontal lines the mean for each experimental group. (B) Kinetics analysis of OVA specific lgG titers in sera of immunized mice on day 0,14 , and $28(n=5)$. (C) Analysis of OVA specific IgG subclasses in sera of immunized mice $(n=5)$. Results are expressed as the last dilution (end point dilution, e.p.d.) giving the double value (OD450 $\mathrm{nm}$ ) of the background value (negative control). Differences in outcome between the group receiving OVA + c-di-AMP co-encapsulated in IMSG NPs and all other tested groups were assessed using nonparametric ANOVA analyses with Kruskal-Wallis test. Differences were considered significant at $p<0.05\left({ }^{*}\right)$, and $p<0.001\left(^{* * *}\right)$, respectively.

\subsubsection{Adjuvanted IMSG NPs stimulate efficient cellular immune responses following TF vaccination}

The strongest cellular responses at local and systemic levels were stimulated by OVA + c-di-AMP co-encapsulated in IMSG NPs, as indicated by strong proliferative responses both at systemic (Fig. 6A upper panel; $p<0.05$ ) and local level (Fig. 6A lower panel; $p<0.01$ ), as compared to cells derived from mice immunized with OVA and Blank NPs.

When analyzing the cytokine profiles stimulated following TF vaccination, it turned out that the application of IMSG NPs on intact skin stimulated high concentrations of IFN $\gamma$, IL-13 and IL-17 and small amounts of IL-5 and IL-10, followed by IL-2, IL-4 and IL-6 (Fig. 6B). However, while at the local level (draining LNs) IFN $\gamma$ was the most prominent cytokine followed by IL-13, IL-17 and IL-5, at the systemic level this pattern 
was only observed for splenocytes from mice receiving OVA + c-di-AMP coencapsulated in IMSG NPs (Fig. 6B). Splenocytes from mice receiving all other formulations produced predominantly IL-13, followed by IFN $\gamma$ and IL-17. In terms of multifunctional T cells, immunization with either OVA alone, OVA co-administered with c-di-AMP or OVA-encapsulated IMSG NPs resulted in the generation of mainly ( 70\%) mono-functional (IFN $\gamma$ ) CD4+ T cells, followed by bi-functional (IFN $\gamma / T N F a$,

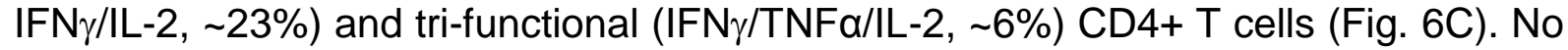
significant differences were observed between these groups. In contrast, mice immunized with OVA + c-di-AMP co-encapsulated IMSG NPs showed an improved quality of CD4+ responses, as indicated by increased proportions of bi- and trifunctional CD4+ cells (57\% and 19\%, respectively; see Fig. 6C). However, only low frequencies of OVA-specific CD8+ T cells were detected as it was observed in the case of IN vaccination, which only showed mono-functional capacities (data not shown).

\subsection{Intradermal vaccination}

\subsubsection{Adjuvanted IMSG NPs stimulate efficient humoral immune responses following ID immunization}

As in the case of IN and TF immunizations, delivery of vaccine formulations via the ID route was well tolerated, since no obvious signs of acute toxicity (e.g. weight loss, reduced mobility, alopecia) were observed. The highest OVA-specific IgG titer was stimulated by OVA + c-di-AMP co-encapsulated IMSG NPs, as compared to Blank $(p<0.01)$ and OVA solution $(p<0.05)$ (Fig. 7A). Interestingly, high lgG titers were observed even after a single boost, whereas for all other formulations, a second boost was needed in order to stimulate antibody production (Fig. 7B).

In contrast to IN and TF vaccine administration, incorporation of c-di-AMP as adjuvant resulted in the stimulation of balanced Th1/Th2 responses, as indicated by increased levels of both IgG1 and IgG2c antibodies in sera of immunized mice (Fig. 7C). In contrast, OVA protein alone or encapsulated in IMSG NPs stimulated predominant production of $\lg \mathrm{G} 1$, indicating a Th2 biased response. 

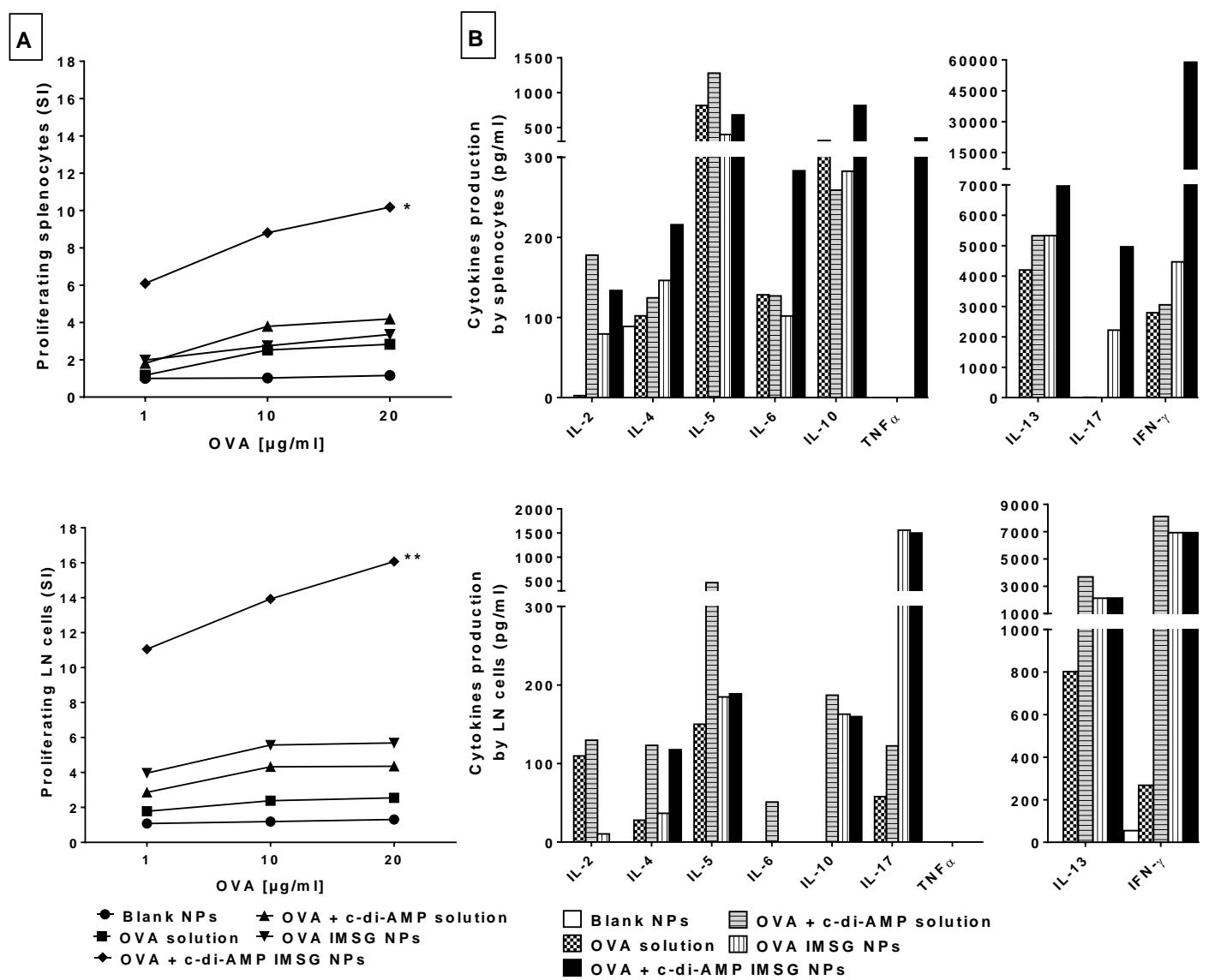

c

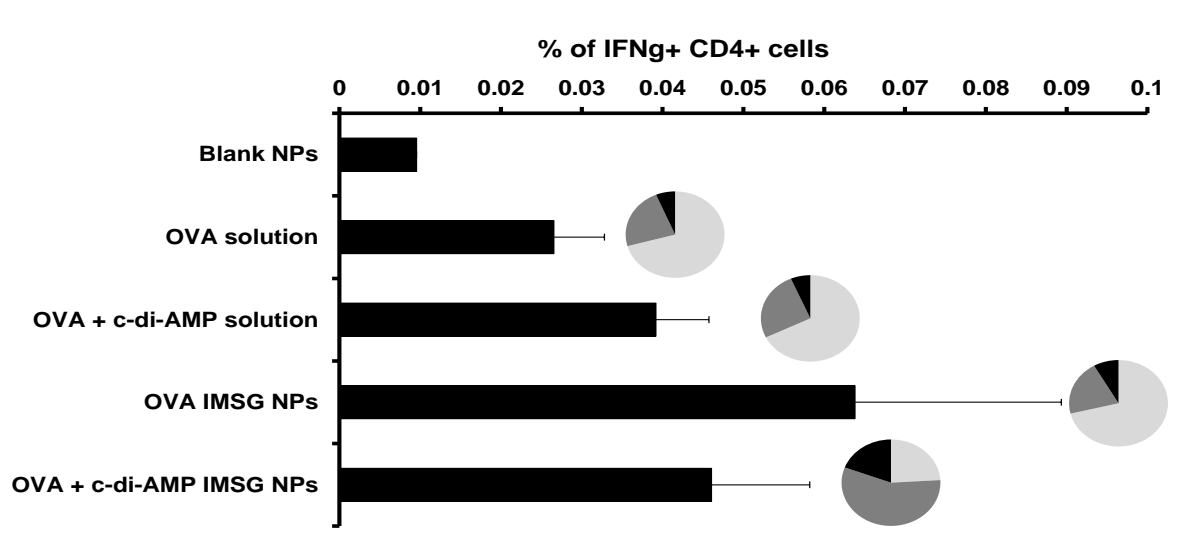

Fig. 6. Cellular responses stimulated following TF immunization. (A and B) Splenocytes (upper panel) and $\mathrm{LN}$ cells (lower panel) from vaccinated animals were restimulated with different concentrations of OVA for $96 \mathrm{~h}$. (A) Cellular proliferation was assessed by determination of the $\left[{ }^{3} \mathrm{H}\right]$ thymidine incorporated into the DNA of replicating cells. Results are averages of quadruplicates and expressed as stimulation index $(\mathrm{SI})$. (B) Cytokine profiles stimulated by the different vaccine formulations were evaluated by cytometric bead array. Results are expressed in $\mathrm{pg} / \mathrm{ml}$. (C) Quality of the $\mathrm{T}$ cell responses stimulated following IN vaccination. Results are expressed as \% of all CD4+ cells acquired per sample (approx. 130,000 CD4+ cells). This subpopulation was further divided into mono-functional (IFN $\gamma$ positive) (light gray), bi-functional (IFN $\gamma /$ IL-2 or IFN $\gamma /$ TNF $\alpha$ positive) (dark gray) and trifunctional (IFN $\gamma$, IL-2 and TNF $\alpha$ positive) (black). The differences were considered significant as compared to values in mice receiving OVA alone at $p<0.05\left(^{*}\right)$ and $p<0.01\left(^{* *}\right)$, respectively. 


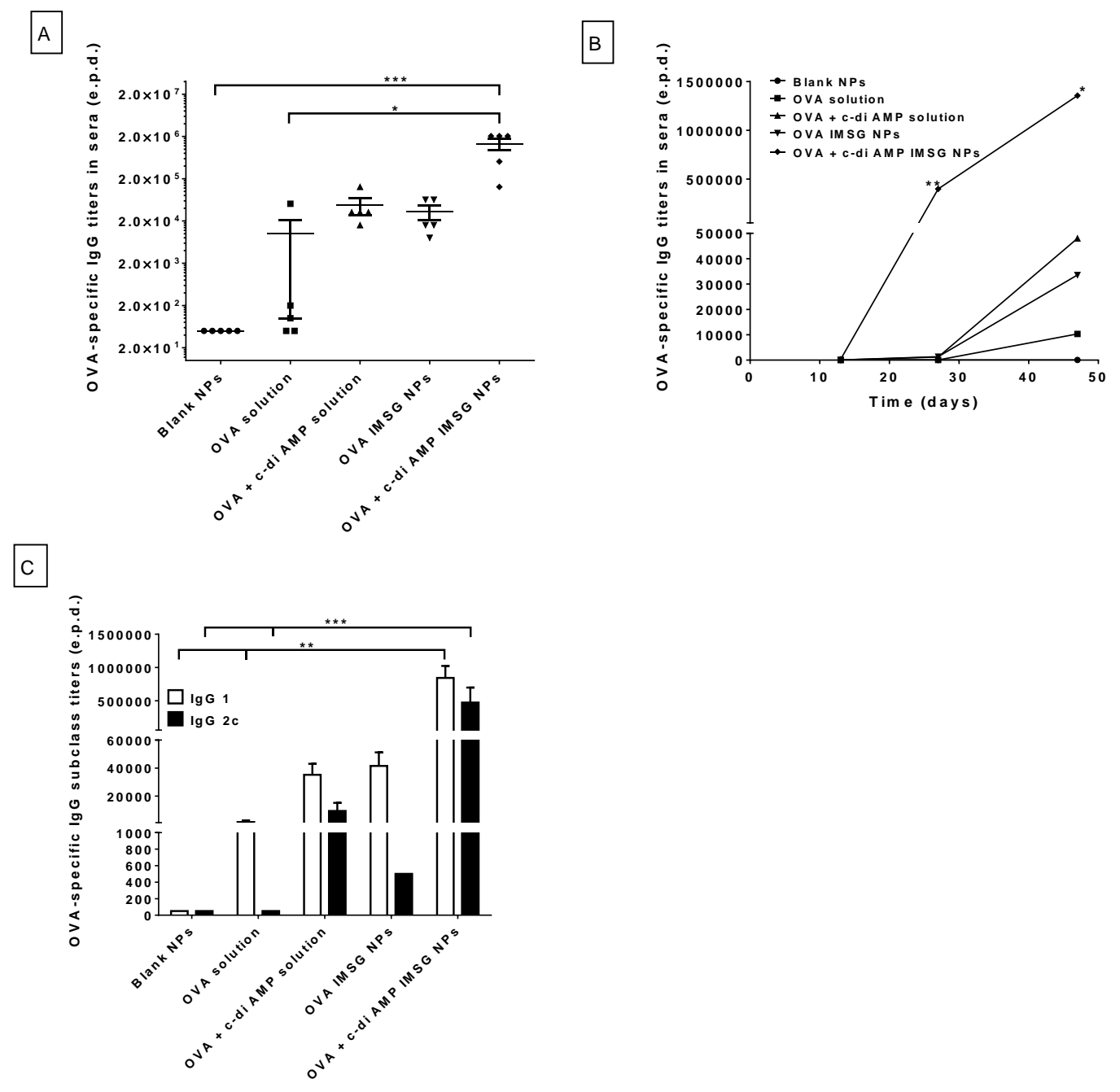

Fig. 7. Systemic humoral immune responses stimulated in C57BL/6 mice $(n=5)$ after three immunizations with different OVA containing formulations by ID route. (A) OVA-specific IgG titers in sera of mice 20 days after the last immunization. Bullet points indicate titers for individual animals and horizontal lines the mean for each experimental group. (B) Kinetics of OVA-specific IgG production in sera of immunized mice on day 0,13, 27 and 48. (C) OVA-specific IgG subclasses in sera of immunized mice. Results are expressed as the last dilution (end point dilution, e.p.d.) giving the double value (OD450 nm) of the background value (negative control). Standard error of mean (SEM) is indicated by vertical lines. Differences were considered significant between the group receiving OVA + c-di-AMP co-encapsulated IMSG NPs and groups receiving OVA solution and Blank at $p<0.05\left(^{*}\right)$, $\mathrm{p}<0.01\left(^{* *}\right)$ and $\mathrm{p}<0.001\left(^{* * *}\right)$, respectively.

\subsubsection{Adjuvanted IMSG NPs stimulate efficient cellular immune responses} following ID immunization

In accordance to the humoral immune responses observed following immunization via the ID route, vaccination of mice using OVA + c-di-AMP co-encapsulated in IMSG NPs stimulated the strongest cellular responses, whereas OVA protein alone or encapsulated in IMSG NPs were less efficient (Fig. 8A). The absolute numbers of proliferating cells were higher at the local level with respect to systemic responses (data not shown). In terms of cytokine release profile, the strongest cytokine production was stimulated in mice immunized with OVA co-administered with c-diAMP and those receiving OVA + C-di-AMP co-encapsulated in IMSG NPs (Fig. 8B). Only vaccine formulations including c-di-AMP stimulated strong production of IFN $\gamma$. OVA alone or OVA encapsulated in IMSG NPs stimulated predominantly IL-13, followed by IL-5 and IL-10 (Fig. 8B). In addition, only mice receiving OVA + C-di-AMP 
co-encapsulated in IMSG NPs showed significantly increased levels of TNF- $\alpha$, IL-17 and IL-6. Besides the fact that the levels of IL-5, IL-13 and INF $\gamma$ seem to be higher in mice receiving OVA-loaded IMSG NPs, the differences were not significant compared to the cytokine profiles of mice receiving the OVA protein alone. In line with the obtained IgG subclass patterns, vaccine formulations containing c-di-AMP stimulated a balanced Th1/Th2 response, whereas those without adjuvant promoted a Th2dominated response (Fig. 8B).

When analyzing the functional spectrum of antigen-specific $T$ cells stimulated following ID immunization, similar results have been obtained compared to TF vaccination. Thus, administration of OVA + c-di-AMP co-encapsulated IMSG NPs via the ID route stimulated the highest number of IFN $\gamma$ producing CD4+ T cells (Fig. $8 \mathrm{C}$ ).
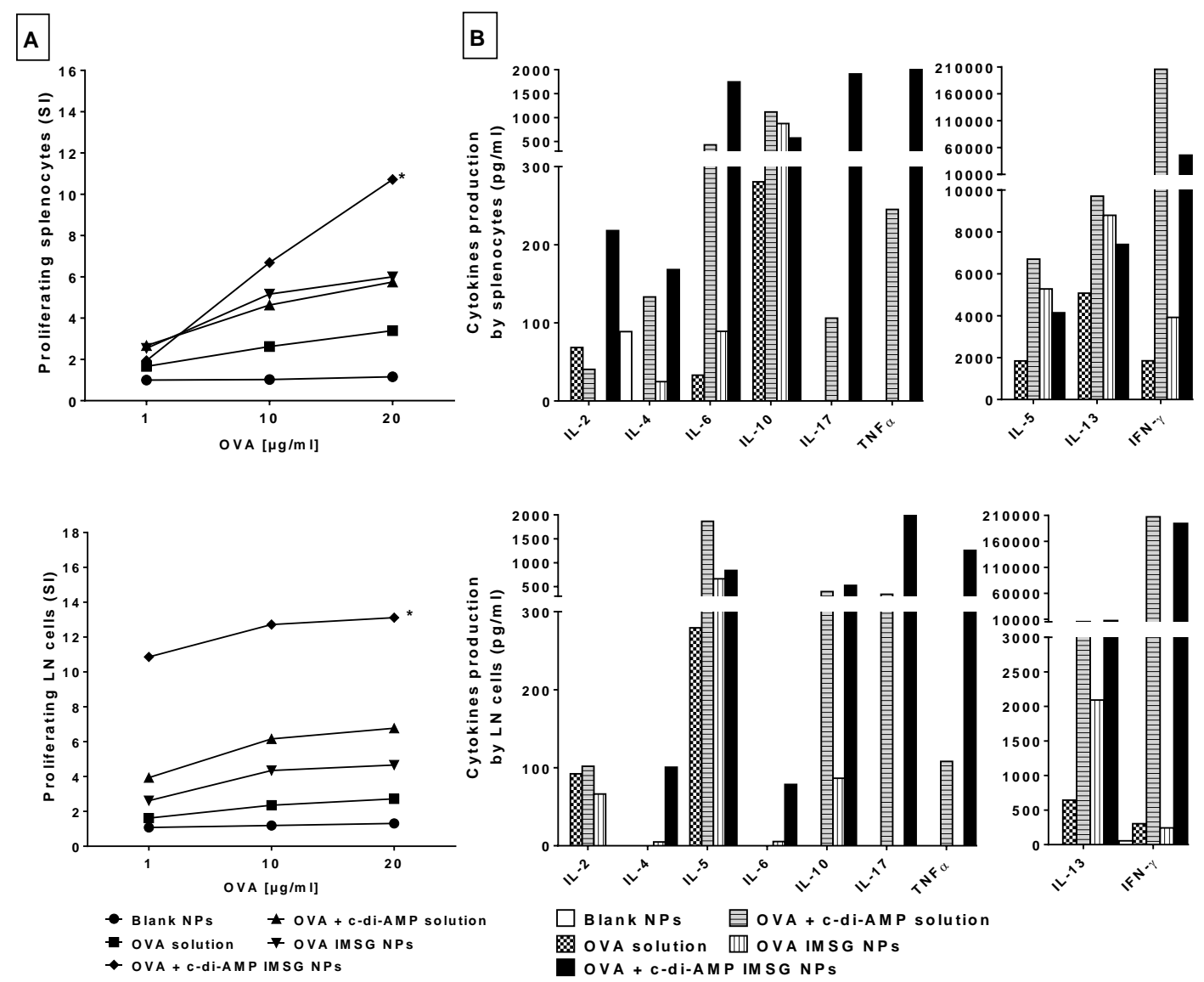

c

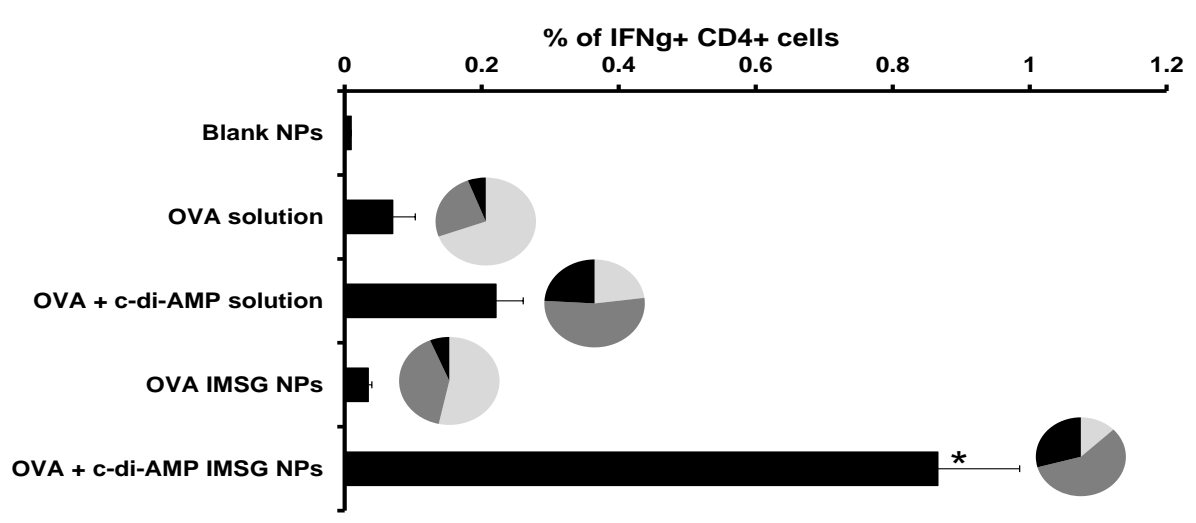

Fig. 8. Cellular responses stimulated following ID immunization. Splenocytes (upper panel) and LN cells (lower panel) from vaccinated animals were re-stimulated with different concentrations of OVA for 
$96 \mathrm{~h}$. (A) Cellular proliferation was assessed by determination of the $\left[{ }^{3} \mathrm{H}\right]$ thymidine incorporated into the DNA of replicating cells. Results are averages of quadruplicates and expressed as stimulation index (SI). (B) Cytokine profiles stimulated by the different vaccine formulations were evaluated by cytometric bead array. Results are expressed in $\mathrm{pg} / \mathrm{ml}$. (C) Quality of the T cell responses stimulated following IN vaccination. Results are expressed as \% of all CD4+ cells acquired per sample (approx. $130,000 \mathrm{CD} 4+$ cells). This subpopulation was further divided into mono-functional (IFN $\gamma$ positive) (light gray), bi-functional (IFN $\gamma /$ IL-2 or IFN $\gamma /$ TNF $\alpha$ positive) (dark gray) and tri-functional (IFN $\gamma$, IL-2 and TNF $\alpha$ positive) (black). The differences were considered significant as compared to values in mice receiving OVA alone at $p<0.05\left(^{*}\right)$.

Moreover, OVA-encapsulated IMSG NPs already increase the proportion of OVAspecific bi-functional (IFN $\gamma / \mathrm{TNF} \alpha$, IFN $\gamma / \mathrm{IL}-2$ ) and tri-functional (IFN $\gamma / T N F \alpha / I L-2)$ CD4+ $\mathrm{T}$ cells, as compared to OVA alone (46\% vs $31 \%)$. This effect was further strengthened (87\%) using OVA + C-di-AMP co-encapsulated IMSG NPs (Fig. 8C). A similar pattern was observed following vaccination with OVA protein admixed with cdi-AMP (77\%). Nevertheless, none of the vaccine formulations seem to be able to stimulate antigen-specific CD8+ T cells showing multifunctional properties (data not shown).

\section{Discussion}

Micro- and nanoparticle based delivery systems offer unique possibilities to create safe and effective vaccines [21]. However, the route of vaccination is also an important parameter to define the immune response triggered by particulate carriers. This is not only due to differences in physicochemical properties (e.g. size, surface charge) of the carrier, but also due to targeted antigen delivery to different subsets of APCs located at the inductive site [22]. Among the various potential routes of immunization, the transcutaneous and nasal routes aroused considerable interest, particularly due to their amenability for non-invasive delivery of vaccines.

Most of the currently studied polymeric NPs are based on PLGA and PLA, but suffer from multiple limitations. Among them can be mentioned the use of chemical solvents and physical stresses during the production process, which negatively affect antigen stability, and the involvement of complex multistep processes to generate them [5]. In the present work we overcome some of the problems described above by using a simple approach based on inverse micelles. IMSG NPs were prepared using pharmaceutical excipients such as MCT, lecithin, span 80 etc. which are GRAS listed, biocompatible and biodegradable. Furthermore, antigen encapsulated in IMSG NPs turned out to be much more immunogenic $(85 \pm 5 \%)$ in comparison to our previously described antigen-loaded chitosan-PLGA NPs (65 $\pm 5 \%$ ) when analyzed using ELISA [12].

Adjuvants play a vital role in vaccine development, as they increase immunogenicity of weak antigens as well as decrease the dose of the antigen required in vaccine formulations [3]. In this study we explored the possibility of improving the performance of the formulations by using c-di-AMP, since preliminary studies suggested that c-di-AMP was compatible with the nanoparticle-based platform. This adjuvant belongs to a new group of natural compounds, the cyclic di-nucleotides, which exhibit strong immune stimulating properties $[15,17,19,20]$. The c-di-AMP enhances dendritic cells (DC) activation, resulting in increase of both antigen-specific humoral and cellular immune responses, which confer protection against infections $[15,17,18]$. STING (stimulator of IFN genes) was identified as an innate sensor of cyclic di-nucleotides. Interaction with STING activates the TBK-IRF3 signaling pathway, leading to the production of type I IFN and TNF $\alpha$ [23-26]. 
We evaluated the antigen delivery efficiency of our new particulate system (IMSG NPs) by immunizing mice via three different routes (TF, IN and ID). Surprisingly, vaccine formulations based on IMSG NPs did not stimulate efficient immune responses when administered via the IN route. IN delivery of OVA-loaded IMSG NPS with or without co-encapsulation of c-di-AMP generated only poor humoral and cellular responses, if any at all. Moreover, an increased proliferative capacity of antigen-specific cells was only observed at the local level. Although we also observed an improved quality of the cellular response, the number of polyfunctional CD4+ T cells was higher using the OVA solution co-administered with c-di-AMP by IN route. Thus, despite the fact that the overall immunogenicity of the IMSG NP-based formulation was rather modest, it was sufficient for priming i.e. differentiation of antigen-specific $T$ and $B$ cells into effector cells. However, factors which might be responsible for the ineffectiveness of the IMSG NPs to act as a delivery system for mucosal administration are (i) NP size [27], (ii) muco-adhesive properties or positive surface charge [28], and (iii) oil viscosity of the NPs [29], as they can hinder the delivery of antigen and adjuvants across the mucosal barrier. Thus, further effort needs to be taken in order to optimize these particles for mucosal applications.

Since our group very recently demonstrated the potential of TF vaccination as a strategy for non-invasive transcutaneous vaccination by topically applying polymeric NPs (Chit-PLGA) without pre-treating the skin [13], we evaluated here IMSG NPs as a delivery system for TF vaccination. For this we evaluated the follicular uptake efficacy of IMSG NPs in a side-by-side comparison with the previously described Chit-PLGA NPs using the excised pig ear model [12]. We observed a significantly higher penetration of OVA-loaded IMSG NPs to the hair follicles compared to the OVA solution and OVA-loaded Chit-PLGA NPs, respectively (Fig. 4). This is in line with results obtained by Raber et al. showing that the penetration of nanoparticles into hair follicles is material dependent [30]. Moreover, using an adoptive $\mathrm{T}$ cell transfer mouse model we further validated the superiority of IMSG NPs compared to Chit-PLGA NPs. We observed an increased induction of OVA-specific $T$ cell proliferation stimulated by OVA-loaded IMSG NPs with respect to the previously evaluated OVA-loaded Chit-PLGA NPs (supplementary Fig. 3) [12]. Therefore, when immunizing mice with OVA-loaded IMSG NPs or OVA + c-di-AMP co-encapsulated IMSG NPs via the TF route, enhanced production of OVA-specific antibodies have been observed compared to OVA alone or OVA + c-di-AMP solution. Moreover, coencapsulation of antigen and adjuvant in the same NP generated the strongest humoral immune response, indicating a synergistic effect of NPs and adjuvant in the formulation that results in strong humoral immune responses.

A similar pattern of the humoral immune response, although to a much higher extent, was also generated by IMSG NPs in the case of ID delivery. This is not surprising since, in contrast to TF immunization, ID application ensures that $100 \%$ of the antigen is delivered to the inductive side, thereby resulting in strong immune responses. These results confirmed the potential of IMSG NPs-based vaccination as an approach to deliver antigens via the ID route. The enhanced humoral response most likely based on the combination of the strong adjuvant activity of c-di-AMP and the depot effect created by the oily character of the IMSG NPs-based system, which in turn ensures antigen and adjuvant release in a sustained manner. Furthermore, co-encapsulation of adjuvant in the NPs significantly boosted the production of the IgG2c subclass, thereby indicating an enhanced Th1 response, which is particularly important in order to efficiently combat intracellular pathogens. In contrast, administration of OVA-encapsulated NPs or soluble OVA admixed with c-di-AMP did not significantly affect the polarization of the antigen-stimulated T-helper cell 
response. This is in line with previous observations demonstrating that coencapsulation of antigen and adjuvant in the same NP is crucial for a potent immune response due to concomitant delivery of antigen and adjuvant to the same APC [22, 31, 32]. Interestingly, although TF vaccination with OVA + c-di-AMP co-encapsulated in IMSG NPs also promoted strong OVA-specific IgG responses, no shift towards a more balanced Th1/Th2 response was observed. This might be explained by the interaction of IMSG NPs with different APCs in different anatomic niches. In the epidermal area of the hair follicle canal mainly LCs are resident, whereas the dermal area is populated by dermal DCs [33]. Both DC subsets were shown to be able to stimulate different, if not opposing, adaptive immune responses. This is not only based on the cytokines produced by these cells following interaction with the antigen and/or adjuvant, but also due to differences in their intrinsic properties, such as cross-presentation [34]. Further studies are needed to elucidate the underlying mechanisms, but several aspects such as miscibility of oil components with sebum and $\mathrm{pH}$ gradient in the skin might play a role. These results were confirmed by the analysis of the cytokines produced by the lymphocytes of immunized mice. Increased cytokine levels, especially of IFN $\gamma$, were observed mainly in mice receiving OVA and c-di-AMP co-encapsulated IMSG NPs and OVA + c-di-AMP solution. Hence, c-diAMP seems to be essential in order to stimulate increased levels of IFN $\gamma$. However, the concurrent expression of the Th1 associated cytokine IFN $\gamma$ and the Th2 associated cytokines IL-13, IL-10 and IL-5 following immunization indicate a balanced Th1/Th2 response, which is in line with the observed IgG subclass profiles. The Th2 biased response observed following TF immunization most likely is owing to the relative low ratio of Th1 and Th2 cytokines compared to the ID and IN routes (reviewed in [35]).

T cell-mediated immunity is a central element of the adaptive immune system, which is needed to eliminate intracellular pathogens, viruses or malignant cells. Thus, we evaluated cellular immune responses following immunization with IMSG NP-based formulations by different routes. Consistent with the humoral responses stimulated after vaccination via the ID and TF routes, OVA + c-di-AMP co-encapsulated within IMSG NPs stimulated the strongest cellular responses, both at the local and systemic level. Interestingly, TF immunization also resulted in enhanced expression of IL-17, whereas vaccination via the ID route did not. This is consistent with observations by Igyártó et al. showing that epidermis-resident LCs promote the stimulation of Th17 cells, whereas dermis-resident DCs rather stimulate Th1 responses and inhibit Th17 differentiation [34]. While Th17 responses were shown to play an important role in host protection against extracellular pathogens (e.g. bacteria and fungi), Th1 responses are needed to efficiently protect against intracellular bacterial and viral infections (reviewed in [36, 37]).

Vaccination using IMSG NPs seems to stimulate only strong CD4+ T helper responses, since only marginal activation of antigen-specific CD8+ T cells could be observed (data not shown). This failure is most likely based on the oily nature of the formulations, as similar observations were made with Freund's incomplete adjuvant (IFA, a water-in-oil emulsion), as well as with oil-in-water emulsions (e.g. MF59 and AS03) [38]. IFA is one of the most commonly used adjuvants in research and is prepared from non-metabolizable oils (paraffin oil and mannide monooleate) lacking the mycobacterial component of complete Freund's adjuvant (CFA), whereas MF59 and AS03 adjuvants encompass squalene, one of the most common lipids produced by human skin cells, forming oil-in-water nano-emulsions made up of droplets less than $250 \mathrm{~nm}$ in size [38, 39]. Both types of adjuvants preferentially polarize the $\mathrm{T}$ cell response towards a functional Th2 phenotype by a yet unknown mechanism [40]. 
Possible explanations can be (i) enhanced antigen uptake by DCs, (ii) reactive oxygen species (ROS)-mediated signaling and (iii) altered cellular lipid metabolism [41-43]. In this regard it was demonstrated that injection of MF59 results in the local release of ATP, which acts as danger signal able to activate the inflammasome by generation of ROS [44-46]. ROS induced in DCs in turn triggers Th2 responses by suppression of Th1 cytokine production, as well as recruitment of IL-4+ basophils [47]. Furthermore, the slow release of antigens by such adjuvants results in a prolonged exposure of immune cells to low antigen levels. This in turn leads to a continued stimulation of $B$ cells, resulting in the production of high antibody levels (reviewed in [48, 49]). In the case of IFA, it has been suggested that NOD2, an intracellular pattern recognition receptor recognizing bacterial components, modulates the adjuvanticity of IFA towards a biased Th2 profile [50]. Incorporation of a potent CD8+ stimulating adjuvant might favor stimulation of IFN $\gamma$ producing CD8+ T cells by IMSG NPs-based formulations. For example, incorporation of the mycobacterial components in CFA results in the stimulation of a Th1 profile (reviewed in [51]). Thus, stimulation of antigen-specific CD8+ T cell responses relies on the signals given to APCs, which result in the interaction of APCs with CD8+ T cells by receptor and cytokine communication. In this regard, administration of extracellular antigens would need to be cross-presented and cytokines, such as IL-2 and TNF $\alpha$, would need to be produced. The mechanism by which cross-presentation is regulated is still unclear. However, while some cells constitutively cross-present antigens, other cells require activation by stimuli (reviewed in [52]). Therefore, the IMSG NPs used in the present work might need to be further optimized in order to deliver the "right" stimuli for activation of the most appropriate DC subset. Another option could be optimizing the antigen concentration, since Ebensen et al. showed efficient stimulation of cytotoxic $\mathrm{T}$ cells after IN immunization by increasing the OVA dosage [15].

There is a consensus that efficient protection against infection not only depends on the number of antigen-specific $T$ cells but also on their functional properties. Thus, there is increasing evidence that $T$ cells which produce several key cytokines at one time are more potent in their effector functions as compared to single producers [5358]. In this regard, the quality of the $T$ helper response stimulated using OVA + C-diAMP in solution by IN and ID routes, or by OVA + c-di-AMP co-encapsulated IMSG NPs delivered by ID and TF routes was significantly improved. Interestingly, while OVA + c-di-AMP solution was able to improve $\mathrm{T}$ cell response quality when mice were immunized by ID and IN routes, this was not the case following TF vaccination. This can be explained by reduced delivery of the vaccine formulation to the hair follicle (as shown in Fig. 4.). However, OVA + c-di-AMP co-encapsulated in IMSG NPs stimulated the highest values of bi- and tri-functional CD4+ T cells following ID and TF immunization. In this regard it is important to consider that in some infections stimulation of multifunctional CD4+ T cells seems to be even more critical to confer protection than activation of CD8+ $\mathrm{T}$ cells $[59,60]$. Taken together, the results presented here underscore the potential of IMSG NPs based vaccine formulations as a technology platform for the delivery of vaccine antigens.

\section{Conclusions}

This study provides further evidence for the potential of the TF route using nanoparticles as a needle-free vaccination approach to deliver antigens through the intact skin. The inherent potential of TF vaccination in conjunction with nanocarriers was demonstrated by the fact that an antigenic solution alone or in combination with an adjuvant applied on intact skin did not generate efficient immune responses. 
IMSG NPs showed enhanced protein encapsulating efficacy, stability and performance than other classical particles, such as PLGA NPs. Co-encapsulation of an adjuvant inside the particles resulted in enhanced antigen-specific humoral and cellular immune responses as compared to non-adjuvanted formulations, when mice were immunized by the ID or TF route,. Besides increasing the strength of the stimulated immune responses, ID and TF immunization of mice with OVA + c-di-AMP co-encapsulated in IMSG NPs also stimulated antigen-specific multifunctional CD4+ $\mathrm{T}$ helper responses with bi- and tri-functional capacity. In addition, the superior immune responses obtained following ID vaccination with antigen and adjuvant coencapsulated in IMSG NPs also illustrate the potential of these particles for the development of new vaccination strategies.

It is also clear that IMSG NPs need to be further optimized as a carrier system for TF vaccines in terms of reducing the required antigen dose and improving the strength of the elicited immune response. In this context, it is reasonable to assume that incremental improvements based on targeting of the nanoparticles to specific subsets of APCs, improvement of their antigen loading capacity and optimization of the vehicle should lead to a significantly enhanced overall efficiency. In the future, efforts should also be undertaken to further validate the efficacy of the immune responses elicited using vaccine relevant antigens.

\section{Acknowledgments}

This work was in part supported by the Bill \& Melinda Gates Foundation (Exploration Grant OPP1015136), A.M. was the recipient of a fellowship from the German Academic Exchange Service (DAAD). We gratefully acknowledge Faerber Fleischgrosshandel (Zweibruecken, Germany) for kind donation of pig ears and UHU $\mathrm{GmbH} \&$ Co. KG for donation of superglue. C. Mathes is thanked for technical assistance for the preparation of the histological sections.

\section{References}

[1] P. Karande, S. Mitragotri, Transcutaneous Immunization: An Overview of Advantages, Disease Targets, Vaccines, and Delivery Technologies, Annu Rev Chem Biomol, 1 (2010) 175-201.

[2] S.M. Bal, Z. Ding, E. van Riet, W. Jiskoot, J.A. Bouwstra, Advances in transcutaneous vaccine delivery: Do all ways lead to Rome?, J Control Release, 148 (2010) 266-282.

[3] A. Mittal, A.S. Raber, C.M. Lehr, S. Hansen, Particle based vaccine formulations for transcutaneous immunization, Hum Vaccin Immunother, 9 (2013) 1950-1955.

[4] A. des Rieux, V. Fievez, M. Garinot, Y.J. Schneider, V. Preat, Nanoparticles as potential oral delivery systems of proteins and vaccines: A mechanistic approach, J Control Release, 116 (2006) 1-27.

[5] B.G. De Geest, M.A. Willart, B.N. Lambrecht, C. Pollard, C. Vervaet, J.P. Remon, J. Grooten, S. De Koker, Surface-Engineered Polyelectrolyte Multilayer Capsules: Synthetic Vaccines Mimicking Microbial Structure and Function, Angew Chem Int Edit, 51 (2012) 3862-3866.

[6] D.J. Irvine, M.A. Swartz, G.L. Szeto, Engineering synthetic vaccines using cues from natural immunity, Nat Mater, 12 (2013) 978-990.

[7] W. Jiskoot, T.W. Randolph, D.B. Volkin, C.R. Middaugh, C. Schoneich, G. Winter, W. Friess, D.J. Crommelin, J.F. Carpenter, Protein instability and immunogenicity: roadblocks to clinical application of injectable protein delivery systems for sustained release, Journal of pharmaceutical sciences, 101 (2012) 946-954.

[8] J. Giri, W.J. Li, R.S. Tuan, M.T. Cicerone, Stabilization of Proteins by Nanoencapsulation in SugarGlass for Tissue Engineering and Drug Delivery Applications, Adv Mater, 23 (2011) 4861-4867.

[9] J.F. Correia-Pinto, N. Csaba, M.J. Alonso, Vaccine delivery carriers: Insights and future perspectives, Int J Pharmaceut, 440 (2013) 27-38.

[10] C.S. Campbell, L.R. Contreras-Rojas, M.B. Delgado-Charro, R.H. Guy, Objective assessment of nanoparticle disposition in mammalian skin after topical exposure, J Control Release, 162 (2012) 201-207. 
[11] B. Mahe, A. Vogt, C. Liard, D. Duffy, V. Abadie, O. Bonduelle, A. Boissonnas, W. Sterry, B. Verrier, U. Blume-Peytavi, B. Combadiere, Nanoparticle-based targeting of vaccine compounds to skin antigen-presenting cells by hair follicles and their transport in mice, J Invest Dermatol, 129 (2009) 1156-1164.

[12] A. Mittal, A.S. Raber, U.F. Schaefer, S. Weissmann, T. Ebensen, K. Schulze, C.A. Guzman, C.M. Lehr, S. Hansen, Non-invasive delivery of nanoparticles to hair follicles: a perspective for transcutaneous immunization, Vaccine, 31 (2013) 3442-3451.

[13] A. Mittal, K. Schulze, T. Ebensen, S. Weissmann, S. Hansen, C.M. Lehr, C.A. Guzman, Efficient nanoparticle-mediated needle-free transcutaneous vaccination via hair follicles requires adjuvantation, Nanomedicine : nanotechnology, biology, and medicine, (2014).

[14] K.A. Traul, A. Driedger, D.L. Ingle, D. Nakhasi, Review of the toxicologic properties of mediumchain triglycerides, Food and chemical toxicology : an international journal published for the British Industrial Biological Research Association, 38 (2000) 79-98.

[15] T. Ebensen, R. Libanova, K. Schulze, T. Yevsa, M. Morr, C.A. Guzman, Bis-(3',5')-cyclic dimeric adenosine monophosphate: strong Th1/Th2/Th17 promoting mucosal adjuvant, Vaccine, 29 (2011) 5210-5220.

[16] R.A. Seder, P.A. Darrah, M. Roederer, T-cell quality in memory and protection: implications for vaccine design (vol 8, pg 247, 2008), Nat Rev Immunol, 8 (2008).

[17] T. Ebensen, K. Schulze, P. Riese, M. Morr, C.A. Guzman, The bacterial second messenger cdiGMP exhibits promising activity as a mucosal adjuvant, Clinical and vaccine immunology : CVI, 14 (2007) 952-958.

[18] M.V. Sanchez, T. Ebensen, K. Schulze, D. Cargnelutti, P. Blazejewska, E.A. Scodeller, C.A. Guzman, Intranasal delivery of influenza rNP adjuvanted with c-di-AMP induces strong humoral and cellular immune responses and provides protection against virus challenge, PLoS One, 9 (2014) e104824.

[19] R. Libanova, T. Ebensen, K. Schulze, D. Bruhn, M. Norder, T. Yevsa, M. Morr, C.A. Guzman, The member of the cyclic di-nucleotide family bis-( $\left.3^{\prime}, 5^{\prime}\right)$-cyclic dimeric inosine monophosphate exerts potent activity as mucosal adjuvant, Vaccine, 28 (2010) 2249-2258.

[20] G.K. Pedersen, T. Ebensen, I.H. Gjeraker, S. Svindland, G. Bredholt, C.A. Guzman, R.J. Cox, Evaluation of the sublingual route for administration of influenza H5N1 virosomes in combination with the bacterial second messenger c-di-GMP, PLoS One, 6 (2011) e26973.

[21] L. Zhao, A. Seth, N. Wibowo, C.X. Zhao, N. Mitter, C.Z. Yu, A.P.J. Middelberg, Nanoparticle vaccines, Vaccine, 32 (2014) 327-337.

[22] B. Slutter, S.M. Bal, Z. Ding, W. Jiskoot, J.A. Bouwstra, Adjuvant effect of cationic liposomes and CpG depends on administration route, J Control Release, 154 (2011) 123-130.

[23] C. Shu, G. Yi, T. Watts, C.C. Kao, P. Li, Structure of STING bound to cyclic di-GMP reveals the mechanism of cyclic dinucleotide recognition by the immune system, Nature structural \& molecular biology, 19 (2012) 722-724.

[24] D.L. Burdette, K.M. Monroe, K. Sotelo-Troha, J.S. Iwig, B. Eckert, M. Hyodo, Y. Hayakawa, R.E. Vance, STING is a direct innate immune sensor of cyclic di-GMP, Nature, 478 (2011) 515-518.

[25] S.M. McWhirter, R. Barbalat, K.M. Monroe, M.F. Fontana, M. Hyodo, N.T. Joncker, K.J. Ishii, S. Akira, M. Colonna, Z.J. Chen, K.A. Fitzgerald, Y. Hayakawa, R.E. Vance, A host type I interferon response is induced by cytosolic sensing of the bacterial second messenger cyclic-di-GMP, J Exp Med, 206 (2009) 1899-1911.

[26] S.M. Blaauboer, V.D. Gabrielle, L. Jin, MPYS/STING-mediated TNF-alpha, not type I IFN, is essential for the mucosal adjuvant activity of $\left(3^{\prime}-5^{\prime}\right)$-cyclic-di-guanosine-monophosphate in vivo, $\mathrm{J}$ Immunol, 192 (2014) 492-502.

[27] M.O. Oyewumi, A. Kumar, Z. Cui, Nano-microparticles as immune adjuvants: correlating particle sizes and the resultant immune responses, Expert review of vaccines, 9 (2010) 1095-1107.

[28] B. Sayin, S. Somavarapu, X.W. Li, M. Thanou, D. Sesardic, H.O. Alpar, S. Senel, Mono-Ncarboxymethyl chitosan (MCC) and $\mathrm{N}$-trimethyl chitosan (TMC) nanoparticles for non-invasive vaccine delivery, Int J Pharm, 363 (2008) 139-148.

[29] F.C. Carvalho, M.S. Barbi, V.H. Sarmento, L.A. Chiavacci, F.M. Netto, M.P. Gremiao, Surfactant systems for nasal zidovudine delivery: structural, rheological and mucoadhesive properties, The Journal of pharmacy and pharmacology, 62 (2010) 430-439.

[30] A.S. Raber, A. Mittal, J. Schafer, U. Bakowsky, J. Reichrath, T. Vogt, U.F. Schaefer, S. Hansen, C.M. Lehr, Quantification of nanoparticle uptake into hair follicles in pig ear and human forearm, Journal of controlled release : official journal of the Controlled Release Society, 179C (2014) 2532.

[31] S. Fischer, E. Schlosser, M. Mueller, N. Csaba, H.P. Merkle, M. Groettrup, B. Gander, Concomitant delivery of a CTL-restricted peptide antigen and CpG ODN by PLGA microparticles induces cellular immune response, J Drug Target, 17 (2009) 652-661. 
[32] J.M. Blander, R. Medzhitov, Toll-dependent selection of microbial antigens for presentation by dendritic cells, Nature, 440 (2006) 808-812.

[33] J.M. Moresi, T.D. Horn, Distribution of Langerhans cells in human hair follicle, J Cutan Pathol, 24 (1997) 636-640.

[34] B.Z. Igyarto, K. Haley, D. Ortner, A. Bobr, M. Gerami-Nejad, B.T. Edelson, S.M. Zurawski, B. Malissen, G. Zurawski, J. Berman, D.H. Kaplan, Skin-Resident Murine Dendritic Cell Subsets Promote Distinct and Opposing Antigen-Specific T Helper Cell Responses, Immunity, 35 (2011) 260-272.

[35] P. Kidd, Th1/Th2 balance: the hypothesis, its limitations, and implications for health and disease, Alternative medicine review : a journal of clinical therapeutic, 8 (2003) 223-246.

[36] S.A. Khader, S.L. Gaffen, J.K. Kolls, Th17 cells at the crossroads of innate and adaptive immunity against infectious diseases at the mucosa, Mucosal Immunol, 2 (2009) 403-411.

[37] F. Broere, S. Apasov, M. Sitkovsky, W. Eden, A2 T cell subsets and T cell-mediated immunity, in: F.P. Nijkamp, M.J. Parnham (Eds.) Principles of Immunopharmacology, Birkhäuser Basel, 2011, pp. 15-27.

[38] A. Podda, The adjuvanted influenza vaccines with novel adjuvants: experience with the MF59adjuvanted vaccine, Vaccine, 19 (2001) 2673-2680.

[39] E. Lindblad, Freund's Adjuvants, in: D. O'Hagan (Ed.) Vaccine Adjuvants, Springer New York, 2000, pp. 49-63.

[40] G. Galli, D. Medini, E. Borgogni, L. Zedda, M. Bardelli, C. Malzone, S. Nuti, S. Tavarini, C. Sammicheli, A.K. Hilbert, V. Brauer, A. Banzhoff, R. Rappuoli, G. Del Giudice, F. Castellino, Adjuvanted $\mathrm{H} 5 \mathrm{~N} 1$ vaccine induces early CD4+ T cell response that predicts long-term persistence of protective antibody levels, Proc Natl Acad Sci U S A, 106 (2009) 3877-3882.

[41] A. Seubert, E. Monaci, M. Pizza, D.T. O'Hagan, A. Wack, The adjuvants aluminum hydroxide and MF59 induce monocyte and granulocyte chemoattractants and enhance monocyte differentiation toward dendritic cells, J Immunol, 180 (2008) 5402-5412.

[42] S. Calabro, M. Tortoli, B.C. Baudner, A. Pacitto, M. Cortese, D.T. O'Hagan, E. De Gregorio, A. Seubert, A. Wack, Vaccine adjuvants alum and MF59 induce rapid recruitment of neutrophils and monocytes that participate in antigen transport to draining lymph nodes, Vaccine, 29 (2011) 18121823.

[43] L. Kalvodova, Squalene-based oil-in-water emulsion adjuvants perturb metabolism of neutral lipids and enhance lipid droplet formation, Biochem Biophys Res Commun, 393 (2010) 350-355.

[44] M. Vono, M. Taccone, P. Caccin, M. Gallotta, G. Donvito, S. Falzoni, E. Palmieri, M. Pallaoro, R. Rappuoli, F. Di Virgilio, E. De Gregorio, C. Montecucco, A. Seubert, The adjuvant MF59 induces ATP release from muscle that potentiates response to vaccination, Proc Natl Acad Sci U S A, 110 (2013) 21095-21100.

[45] C.M. Cruz, A. Rinna, H.J. Forman, A.L. Ventura, P.M. Persechini, D.M. Ojcius, ATP activates a reactive oxygen species-dependent oxidative stress response and secretion of proinflammatory cytokines in macrophages, The Journal of biological chemistry, 282 (2007) 2871-2879.

[46] S.L. Cassel, S. Joly, F.S. Sutterwala, The NLRP3 inflammasome: a sensor of immune danger signals, Seminars in immunology, 21 (2009) 194-198.

[47] H. Tang, W. Cao, S.P. Kasturi, R. Ravindran, H.I. Nakaya, K. Kundu, N. Murthy, T.B. Kepler, B. Malissen, B. Pulendran, The T helper type 2 response to cysteine proteases requires dendritic cell-basophil cooperation via ROS-mediated signaling, Nature immunology, 11 (2010) 608-617.

[48] N. Petrovsky, J.C. Aguilar, Vaccine adjuvants: current state and future trends, Immunology and cell biology, 82 (2004) 488-496.

[49] H.F. Stills, Jr., Adjuvants and antibody production: dispelling the myths associated with Freund's complete and other adjuvants, ILAR journal / National Research Council, Institute of Laboratory Animal Resources, 46 (2005) 280-293.

[50] L.O. Moreira, A.M. Smith, A.A. DeFreitas, J.E. Qualls, K.C. El Kasmi, P.J. Murray, Modulation of adaptive immunity by different adjuvant-antigen combinations in mice lacking Nod2, Vaccine, 26 (2008) 5808-5813.

[51] A. Billiau, P. Matthys, Modes of action of Freund's adjuvants in experimental models of autoimmune diseases, Journal of leukocyte biology, 70 (2001) 849-860.

[52] W.R. Heath, G.T. Belz, G.M. Behrens, C.M. Smith, S.P. Forehan, I.A. Parish, G.M. Davey, N.S. Wilson, F.R. Carbone, J.A. Villadangos, Cross-presentation, dendritic cell subsets, and the generation of immunity to cellular antigens, Immunological reviews, 199 (2004) 9-26.

[53] R.A. Seder, P.A. Darrah, M. Roederer, T-cell quality in memory and protection: implications for vaccine design, Nat Rev Immunol, 8 (2008) 247-258.

[54] J.R. Almeida, D.A. Price, L. Papagno, Z.A. Arkoub, D. Sauce, E. Bornstein, T.E. Asher, A. Samri, A. Schnuriger, I. Theodorou, D. Costagliola, C. Rouzioux, H. Agut, A.G. Marcelin, D. Douek, B. 
Autran, V. Appay, Superior control of HIV-1 replication by CD8+ T cells is reflected by their avidity, polyfunctionality, and clonal turnover, J Exp Med, 204 (2007) 2473-2485.

[55] P.A. Darrah, S.T. Hegde, D.T. Patel, R.W. Lindsay, L. Chen, M. Roederer, R.A. Seder, IL-10 production differentially influences the magnitude, quality, and protective capacity of Th1 responses depending on the vaccine platform, J Exp Med, 207 (2010) 1421-1433.

[56] P.A. Darrah, D.T. Patel, P.M. De Luca, R.W. Lindsay, D.F. Davey, B.J. Flynn, S.T. Hoff, P. Andersen, S.G. Reed, S.L. Morris, M. Roederer, R.A. Seder, Multifunctional TH1 cells define a correlate of vaccine-mediated protection against Leishmania major, Nature medicine, 13 (2007) 843-850.

[57] F. Graw, R.R. Regoes, Predicting the impact of CD8+ T cell polyfunctionality on HIV disease progression, Journal of virology, 88 (2014) 10134-10145.

[58] S. Kannanganat, C. Ibegbu, L. Chennareddi, H.L. Robinson, R.R. Amara, Multiple-cytokineproducing antiviral CD4 T cells are functionally superior to single-cytokine-producing cells, Journal of virology, 81 (2007) 8468-8476.

[59] S.G. Morrison, H. Su, H.D. Caldwell, R.P. Morrison, Immunity to murine Chlamydia trachomatis genital tract reinfection involves B cells and CD4(+) T cells but not CD8(+) T cells, Infect Immun, 68 (2000) 6979-6987.

[60] D.A. Kaveh, V.S. Bachy, R.G. Hewinson, P.J. Hogarth, Systemic BCG immunization induces persistent lung mucosal multifunctional CD4 T(EM) cells which expand following virulent mycobacterial challenge, PLoS One, 6 (2011) e21566. 


\section{Figure caption}

Figure 1. TEM image of OVA-loaded IMSG NPS

Figure 2. Systemic humoral immune responses stimulated in C57BL/6 mice $(n=5)$ after three immunizations with different OVA containing formulations via the intranasal (IN) route. (A) OVAspecific $\lg G$ titers in sera of mice 20 days after the last immunization. Bullet points indicate titers for individual animals and horizontal lines the mean for each experimental group. (B) Kinetics of OVAspecific IgG production in sera of immunized mice on day 0,13, 27 and 48. (C) OVA-specific IgG subclasses in sera of immunized mice. Results are expressed as the last dilution (end point dilution, e.p.d.) giving the double value (OD450 nm) of the background value (negative control). Standard error of mean (SEM) is indicated by vertical lines. Differences between the group receiving OVA + C-di-AMP solution and all other tested groups were considered significant at $p<0.05\left(^{*}\right)$, and $p<0.01\left(^{* *}\right)$, respectively.

Figure 3. Cellular responses stimulated following IN immunization. (A and B) Splenocytes (upper panel) and LN cells (lower panel) from vaccinated animals were restimulated with different concentrations of OVA for $96 \mathrm{~h}$. (A) Cellular proliferation was assessed by determination of the $\left[{ }^{3} \mathrm{H}\right]$ thymidine incorporated into the DNA of replicating cells. Results are averages of quadruplicates and expressed as stimulation index (SI). (B) Cytokine profiles stimulated by the different vaccine formulations were evaluated by cytometric bead array. Results are expressed in $\mathrm{pg} / \mathrm{ml}$. (C) Quality of the $T$ cell responses stimulated following IN vaccination. Results are expressed as \% of all CD4+ cells acquired per sample (approx. 130,000 CD4+ cells). This subpopulation was further divided into monofunctional (IFN $\gamma$ positive) (light gray), bi-functional (IFN $\gamma$ / IL-2 or IFN $\gamma$ / TNF $\alpha$ positive) (dark gray) and tri-functional cells (IFN $\gamma$, IL-2 and TNF $\alpha$ positive) (black). Differences were considered significant as compared to values in mice receiving OVA alone at $\left.p<0.01{ }^{* \star}\right)$ and $\left.p<0.001{ }^{* \star *}\right)$, respectively.

Figure 4. A) Distribution of fluorescently labeled NPs in hair follicles after application to excised pig ears. (LSM 510 Meta, Carl Zeiss GmbH, Jena Germany; excitation wavelength $458 \mathrm{~nm}$ ), Transversal cryo-section (thickness $12 \mu \mathrm{m})(B)$; overlay of fluorescence and transmission light images (C): NPs are visible on the skin surface and inside the follicle. (D) Uptake of FITC-labeled OVA encapsulated in IMSG NPs relative to FITC-OVA solution and FITC-OVA in CP NPs. $(n=6$, mean \pm SD; differences were considered significant whenever $\left.p<0.05\left({ }^{*}\right), p<0.001{ }^{(* *}\right)$ compared to OVA-FITC solution.)

Figure 5. Humoral immune responses stimulated in C57BL/6 mice after three vaccinations with OVA containing formulations by TF route. (A) Total OVA specific IgG titers in sera after immunization $(n=$ 5). Bullet points indicate titers for individual animals and horizontal lines the mean for each experimental group. (B) Kinetics analysis of OVA specific IgG titers in sera of immunized mice on day 0,14 , and $28(n=5)$. (C) Analysis of OVA specific IgG subclasses in sera of immunized mice $(n=5)$. Results are expressed as the last dilution (end point dilution, e.p.d.) giving the double value (OD450 $\mathrm{nm}$ ) of the background value (negative control). Differences in outcome between the group receiving OVA + C-di-AMP co-encapsulated in IMSG NPs and all other tested groups were assessed using nonparametric ANOVA analyses with Kruskal-Wallis test. Differences were considered significant at $p<0.05\left(^{*}\right)$, and $p<0.001\left(^{* * *}\right)$, respectively. 
Figure 6. Cellular responses stimulated following TF immunization. (A and B) Splenocytes (upper panel) and LN cells (lower panel) from vaccinated animals were restimulated with different concentrations of OVA for $96 \mathrm{~h}$. (A) Cellular proliferation was assessed by determination of the [ $\left.{ }^{3} \mathrm{H}\right]$ thymidine incorporated into the DNA of replicating cells. Results are averages of quadruplicates and expressed as stimulation index (SI). (B) Cytokine profiles stimulated by the different vaccine formulations were evaluated by cytometric bead array. Results are expressed in $\mathrm{pg} / \mathrm{ml}$. (C) Quality of the T cell responses stimulated following IN vaccination. Results are expressed as \% of all CD4+ cells acquired per sample (approx. 130,000 CD4+ cells). This subpopulation was further divided into monofunctional (IFN $\gamma$ positive) (light gray), bi-functional (IFN $\gamma$ / IL-2 or IFN $\gamma$ / TNF $\alpha$ positive) (dark gray) and tri-functional (IFN $\gamma$, IL-2 and TNF $\alpha$ positive) (black). The differences were considered significant as compared to values in mice receiving OVA alone at $p<0.05\left(^{*}\right)$ and $p<0.01\left(^{* *}\right)$, respectively.

Figure 7. Systemic humoral immune responses stimulated in C57BL/6 mice $(n=5)$ after three immunizations with different OVA containing formulations by ID route. (A) OVA-specific IgG titers in sera of mice 20 days after the last immunization. Bullet points indicate titers for individual animals and horizontal lines the mean for each experimental group. (B) Kinetics of OVA-specific IgG production in sera of immunized mice on day $0,13,27$ and 48. (C) OVA-specific IgG subclasses in sera of immunized mice. Results are expressed as the last dilution (end point dilution, e.p.d.) giving the double value (OD450 nm) of the background value (negative control). Standard error of mean (SEM) is indicated by vertical lines. Differences were considered significant between the group receiving OVA + c-di-AMP co-encapsulated IMSG NPs and groups receiving OVA solution and Blank at $p<0.05\left({ }^{*}\right)$, $p<0.01\left(^{* *}\right)$ and $p<0.001\left(^{* * *}\right)$, respectively.

Figure 8. Cellular responses stimulated following ID immunization. Splenocytes (upper panel) and LN cells (lower panel) from vaccinated animals were re-stimulated with different concentrations of OVA for $96 \mathrm{~h}$. (A) Cellular proliferation was assessed by determination of the $\left[{ }^{3} \mathrm{H}\right]$ thymidine incorporated into the DNA of replicating cells. Results are averages of quadruplicates and expressed as stimulation index (SI). (B) Cytokine profiles stimulated by the different vaccine formulations were evaluated by cytometric bead array. Results are expressed in $\mathrm{pg} / \mathrm{ml}$. (C) Quality of the T cell responses stimulated following IN vaccination. Results are expressed as \% of all CD4+ cells acquired per sample (approx. 130,000 CD4+ cells). This subpopulation was further divided into mono-functional (IFN $\gamma$ positive) (light gray), bi-functional (IFN / IL-2 or IFN / TNF $\alpha$ positive) (dark gray) and tri-functional (IFN $\gamma$, IL-2 and TNF $\alpha$ positive) (black). The differences were considered significant as compared to values in mice receiving OVA alone at $p<0.05\left(^{*}\right)$. 
IMSG

Nanoparticle

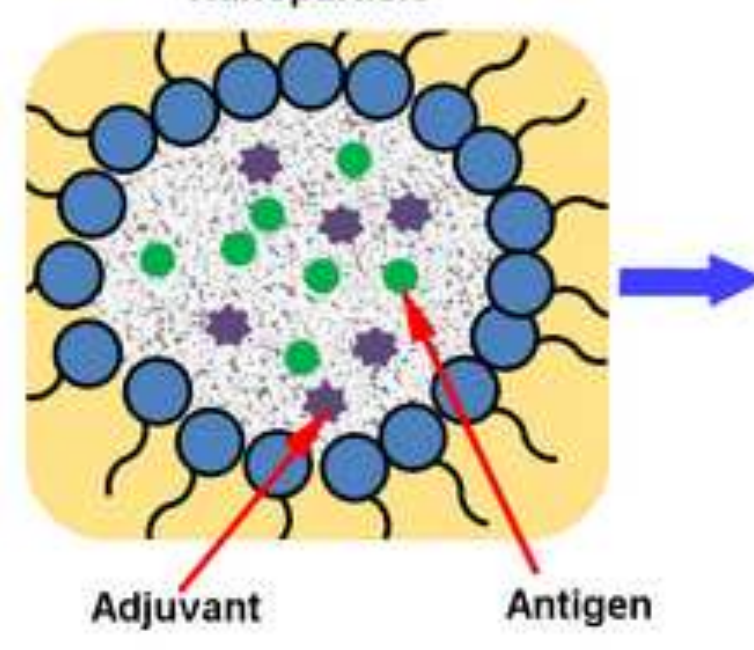

transfollicular

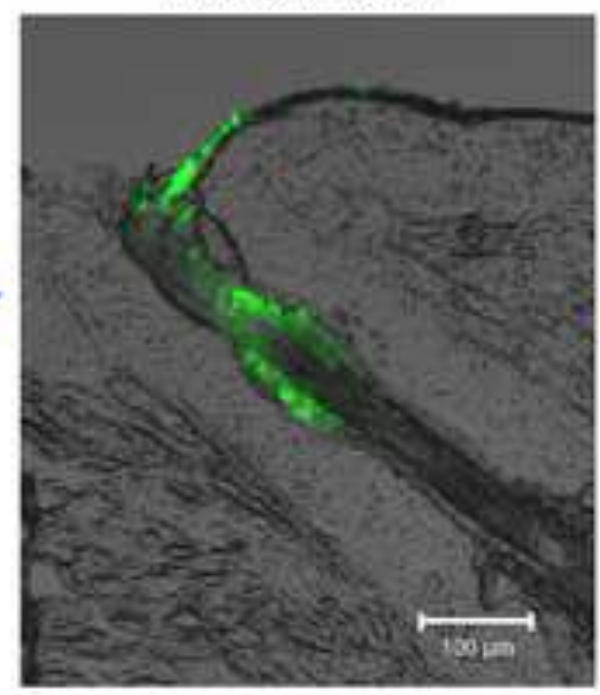

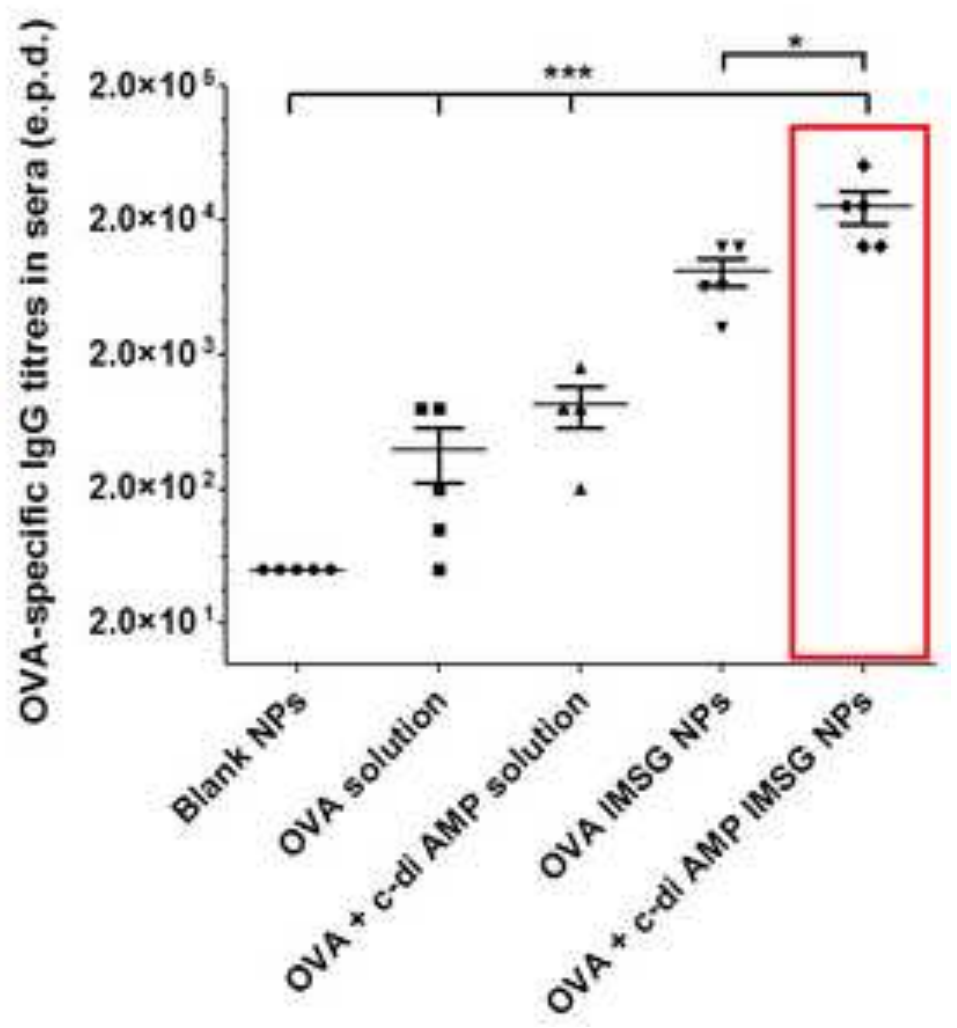

